\title{
ON THE $l$-FUNCTION AND THE REDUCED VOLUME OF PERELMAN I
}

\author{
RUGANG YE
}

\begin{abstract}
The main purpose of this paper is to present a number of analytic and geometric properties of the $l$-function and the reduced volume of Perelman, including in particular the monotonicity, the upper bound and the rigidities of the reduced volume.
\end{abstract}

\section{INTRODUCTION}

In [P1], Perelman introduced, among other things, two important tools for analyzing the Ricci flow: the reduced distance, i.e. the $l$-function, and the reduced volume. The $l$-function is defined in terms of a natural curve energy along the Ricci flow, which is analogous to the classical curve energy employed in the study of geodesics, but involves the evolving metric, as well as the scalar curvature, as a potential term. The reduced volume is a certain integral involving the $l$-function. The $l$-function and the reduced volume enjoy a number of very nice analytic and geometric properties, including in particular the fundamental monotonicity of the reduced volume. These properties can be used, as demonstrated by Perelman, to classify and analyze blow-up limits of the Ricci flow, and to obtain various estimates for the Ricci flow, such as noncollapsing estimates and curvature estimates.

The main purpose of this paper is to present a number of analytic and geometric properties of the $l$-function and the reduced volume, including in particular the monotonicity, the upper bound and the rigidities of the reduced volume. In Perelman's paper, a general assumption concerning the $l$-function and the reduced volume is uniformly bounded sectional curvature. The results obtained in [P1] under this assumption are sufficient for the application to the geometrization of 3 -manifolds in [P2]. Because of the fundamental role of the $l$-function and the reduced volume for analyzing the Ricci flow in general, it is very desirable to allow weaker geometric conditions. Our main focus is to deal with the situation in which only a lower bound for the Ricci curvature is assumed. On the other hand, we hope that our treatment can provide assistance for understanding Perelman's theory, even when one is only interested in the case of bounded sectional curvature.

For the convenience of the reader, we give here a short account of the main topics in this paper. In Section 2, we first present the basic concepts such as the $l$-function, the $\mathcal{L}$-geodesics, and the $\mathcal{L}$-exponential map. Their basic properties are then analysed, which include in particular the local Lipschitz properties and the local semi-concavity of the $l$-function. Several basic estimates for the $l$-function are

Received by the editors May 20, 2006 and, in revised form, September 1, 2006.

2000 Mathematics Subject Classification. Primary 53C20, 53C21. 
also presented. One highlight of this section is Theorem 2.23, which establishes the fundamental differential inequalities (2.73) and (2.74) everywhere in the weak sense, which are formulated in (2.75) and (2.76). Another highlight is the Harnack inequality for the $l$-function in Theorem 2.18.

In Section 3, we first present an estimate for the minimum value of the $l$-function. Next we present an estimate of Perelman which provides a lower bound for the $l$-function at any time in terms of the squared distance at the same time from a fixed point, assuming a nonnegative curvature operator; see Lemma 3.2. (A corresponding upper bound is also included.) In comparison, the easier Lemma 2.3 provides a similar estimate in terms of the squared distance at an earlier time (assuming a lower bound for the Ricci curvature) or a later time (assuming an upper bound for the Ricci curvature). Perelman's estimate provides an important analytic ingredient for dealing with some crucial and delicate convergence issues of integrals involving the $l$-function. Another topic in this section is Theorem 3.3, which expands the scope of Theorem 2.23 to admit test functions which may not have compact support but satisfy a certain decay condition. Such expansions are needed for applying the differential inequalities (2.73) and (2.74) to topics around the reduced volume, as in Section 4 and [Y2].

In Section 4, we derive a number of important properties of the reduced volume, which include the monotonicity (Theorem 4.5), the upper bound (Theorem 4.3), and the rigidity regarding the upper bound (Theorem 4.4). We also derive the rigidity regarding the monotonicity (Theorem 4.9), which says that a solution of the backward Ricci flow must be a gradient shrinking soliton if the values of the reduced volume are equal at two different times. This theme appears again in [Y2] in a different setup.

Communications with Perelman were of great help for understanding his ideas. We also benefited much from conversations with Guofang Wei. We would like to thank Vitali Kapovich for helping to find the reference [GW].

This paper is part of [Y1], whose first version was posted on the author's webpage in February 2004.

\section{BASIC PROPERTIES OF THE $l$-FUNCTION I}

Consider a smooth solution $g=g(\tau)$ of the backward Ricci flow

$$
\frac{\partial g}{\partial \tau}=2 R i c
$$

on a manifold $M$ over an interval $[0, T)$. We assume that $g(\tau)$ is complete for each $\tau \in[0, T)$. Note that the theory presented here is meant to be applied to solutions of the Ricci flow. Indeed, a solution of the Ricci flow can be converted into a solution of the backward Ricci flow by a time reversal.

Notation. We shall denote the distance between two points $q_{1}, q_{2}$ with respect to the metric $g(\tau)$ by $d\left(q_{1}, q_{2}, \tau\right), d_{g}\left(q_{1}, q_{2}, \tau\right)$ or $d_{g(\tau)}\left(q_{1}, q_{2}\right)$. The closed geodesic ball of center $q$ and radius $r$ with respect to the metric $g(\tau)$ will be denoted by $B_{r}(q, \tau)$. The volume form of $g(\tau)$ will be denoted by $d q$ or $\left.d q\right|_{\tau}$. The scalar curvature $R_{g(\tau)}$ of $g(\tau)$ at a point $q$ will be written as $R(q, \tau)$. Similar notation is also used for other curvature quantities. 
An obvious basic lemma is this.

Lemma 2.1. If Ric $\geq-c g$ for a nonnegative constant $c$ on the time interval $[0, \tau]$, then

$$
e^{-2 c s} g(0) \leq g(s) \leq e^{2 c(\tau-s)} g(\tau)
$$

for $s \in[0, \tau]$. If Ric $\leq C g$ for a nonnegative constant $C$ on $[0, \tau]$, then

$$
e^{2 C(s-\tau)} g(\tau) \leq g(s) \leq e^{2 C s} g(0)
$$

for $s \in[0, \tau]$.

We consider Perelman's $\mathcal{L}$-energy for piecewise $C^{1}$ curves $\gamma:[a, b] \rightarrow M, 0 \leq a<$ $b<T$ :

$$
\mathcal{L}_{a, b}(\gamma)=\int_{a}^{b} \sqrt{s}\left(R(\gamma(s), s)+|\dot{\gamma}|^{2}\right) d s,
$$

where $|\cdot|=|\cdot|_{g(s)}$. For a given $\tau$ we abbreviate $\mathcal{L}_{0, \tau}$ to $\mathcal{L}$. The $\mathcal{L}_{a, b}$-geodesic (or $\mathcal{L}$-geodesic) equation is:

$$
\nabla_{\frac{d}{d s}} \dot{\gamma}-\frac{1}{2} \nabla R+\frac{1}{2 s} \dot{\gamma}+2 \operatorname{Ric}(\dot{\gamma}, \cdot)=0,
$$

where $R=R_{g(s)}, R i c=R i c_{g(s)}$, and $\nabla$ is the Levi-Civita connection of $g(s)$. This is the Euler-Lagrange equation of the $\mathcal{L}$-energy. Its (smooth) solutions are called $\mathcal{L}_{a, b}$-geodesics or $\mathcal{L}$-geodesics.

To better understand the properties of $\mathcal{L}_{a, b}$-geodesics, it is helpful to introduce a convenient reparametrization. We set $t=\sqrt{s}$ and $\gamma^{\prime}=d \gamma / d t=2 t \dot{\gamma}$. Then

$$
\mathcal{L}_{a, b}(\gamma)=\int_{\sqrt{a}}^{\sqrt{b}}\left(\frac{1}{2}\left|\gamma^{\prime}\right|^{2}+2 R t^{2}\right) d t,
$$

and the $\mathcal{L}_{a, b}$-geodesic equation becomes

$$
\nabla_{\frac{d}{d t}} \gamma^{\prime}-2 t^{2} \nabla R+4 t \operatorname{Ric}\left(\gamma^{\prime}, \cdot\right)=0
$$

Next we choose a reference point $p \in M$ and define $L(q, \tau)=L_{g}(q, \tau)$ to be the infimum of $\mathcal{L}(\gamma)$ for $\gamma:[0, \tau] \rightarrow M$ with $\gamma(0)=p$ and $\gamma(\tau)=q$. (We write $L_{g}(q, \tau)$ if we need to indicate the dependence on the solution $g$.)

Definition 1. We define the reduced distance (of Perelman) to be

$$
l(q, \tau)=l_{g}(q, \tau)=\frac{L(q, \tau)}{2 \sqrt{\tau}} .
$$

We also call it the $l$-function (of Perelman). The reference point $p$ will be called an l-base.

An easy computation leads to the following basic lemma.

Lemma 2.2. The l-function is invariant under the rescaling $g(\tau) \rightarrow g_{a}(\tau) \equiv$ $a^{-1} g(a \tau)$, i.e.

$$
l_{g_{a}}(q, \tau)=l_{g}(q, a \tau)
$$

for all $\tau \in\left(0, \frac{T}{a}\right)$ and $q \in M$.

Next we derive an estimate for $l$ in terms of the distance function. 
Lemma 2.3. Assume that Ric $\geq-c g$ on $[0, \bar{\tau}]$ for a nonnegative constant $c$. Then

$$
l(q, \tau) \geq e^{-2 c \tau} \frac{d^{2}(p, q, 0)}{4 \tau}-\frac{n c}{3} \tau
$$

for each $\tau \in[0, \bar{\tau}]$. If we assume instead that Ric $\leq C g$ on $[0, \bar{\tau}]$ for a nonnegative constant $C$, then

$$
l(q, \tau) \leq e^{2 C \tau} \frac{d^{2}(p, q, 0)}{4 \tau}+\frac{n C}{3} \tau
$$

for each $\tau \in[0, \bar{\tau}]$.

Proof. We first assume a lower bound on the Ricci curvature. By (2.2) and (2.6) we have, for an arbitrary $\gamma$ from $p$ to $q$,

$$
\mathcal{L}(\gamma) \geq \frac{e^{-2 c \tau}}{2} \int_{0}^{\sqrt{\tau}}\left|\gamma^{\prime}\right|_{g(0)}^{2} d t-\frac{2 n c}{3} \tau^{\frac{3}{2}} \geq e^{-2 c \tau} \frac{d^{2}(p, q, 0)}{2 \sqrt{\tau}}-\frac{2 n c}{3} \tau^{\frac{3}{2}}
$$

This leads to (2.10).

The case of an upper bound for the Ricci curvature is similar, in which we use (2.3) instead of (2.2).

Next we consider Perelman's $\mathcal{L}$-exponential map.

Definition 2. The $\mathcal{L}$-exponential map $\exp _{p}^{\mathcal{L}, \tau}: T_{p} M \rightarrow M$ at time $\tau \in[0, T)$ is defined as follows. For $v \in T_{p} M$, let $\gamma_{v}$ denote the $\mathcal{L}$-geodesic such that $\gamma_{v}(0)=$ $p, \lim _{s \rightarrow 0} \sqrt{s} \dot{\gamma}(s)=v$ (equivalently, $\gamma_{v}^{\prime}(0)=2 v$ ). If $\gamma_{v}$ exists on $[0, \tau]$, we set $\exp _{p}^{\mathcal{L}, \tau}(v)=\gamma_{v}(\tau)$. Let $\mathcal{U}(\tau)$ denote the maximal domain of $\exp _{p}^{\mathcal{L}, \tau}$. By $(2.7)$ and basic ODE, $\mathcal{U}(\tau)$ is an open set and $\exp _{p}^{\mathcal{L}, \tau}$ is a smooth map from $\mathcal{U}(\tau)$ into $M$.

We also have the following extension of the concept of $\mathcal{L}$-exponential map.

Definition 3. For a given reference point $\bar{p}$ and $0<\varepsilon<\tau$ the $\mathcal{L}_{\varepsilon, \tau}$-exponential map $\exp _{\bar{p}} \mathcal{L}_{\varepsilon, \tau}$ is defined as follows. For $v \in T_{\bar{p}} M$, let $\gamma_{v, \varepsilon}$ denote the $\mathcal{L}_{\varepsilon, \tau}$-geodesic such that $\gamma_{v, \varepsilon}(\varepsilon)=\bar{p}, \sqrt{\varepsilon} \dot{\gamma}(\varepsilon)=v$ (equivalently, $\gamma_{v, \varepsilon}^{\prime}=2 v$ at $t=\sqrt{\varepsilon}$ ). If $\gamma_{\varepsilon, v}$ exists on $[0, \tau]$, we set $\exp _{\bar{p}} \mathcal{L}_{\varepsilon, \tau}(v)=\gamma_{\varepsilon, v}(\tau)$.

Proposition 2.4. Assume that the sectional curvature is bounded on $[0, \bar{\tau}]$ for $\bar{\tau} \in(0, T)$. Then $\mathcal{U}(\tau)=T_{p} M$ for each $\tau \in(0, \bar{\tau})$. A similar statement holds true

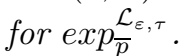

Proof. By the local interior estimates in $[\mathrm{S}]$, the sectional curvature bound on $[0, \bar{\tau}]$ implies an upper bound on $|\nabla R|$ on $[0, \tau]$ for each $\tau \in(0, \bar{\tau})$. Fix $\tau \in(0, \bar{\tau})$ and let $K$ denote an upper bound for $|R i c|$ and $|\nabla R|$ on $M \times[0, \tau]$.

Consider an $\mathcal{L}$-geodesic $\gamma$ with initial time 0 , defined on its maximal interval. We derive from (2.7),

$$
\frac{d}{d t}\left|\gamma^{\prime}\right|^{2}=\frac{\partial g}{\partial s}\left(\gamma^{\prime}, \gamma^{\prime}\right) \frac{d s}{d t}+2 \gamma^{\prime} \cdot \nabla_{\frac{d}{d t}} \gamma^{\prime}=4 t^{2} \nabla R \cdot \gamma^{\prime}
$$

Consequently, we obtain for $t \leq \sqrt{\tau}$ (as long as $\gamma$ is defined)

$$
\left.\left|\frac{d}{d t}\right| \gamma^{\prime}\right|^{2}\left|\leq 4 K t^{2}\right| \gamma^{\prime} \mid
$$

and hence

$$
\left|\frac{d}{d t}\right| \gamma^{\prime}|| \leq 2 K t^{2}
$$


It follows that

$$
\left|\gamma^{\prime}\right| \leq\left|\gamma^{\prime}\right|(0)+\frac{2}{3} K t^{3}
$$

By (2.2) we then infer

$$
\left|\gamma^{\prime}(t)\right|_{g(0)} \leq e^{K t}\left(\left|\gamma^{\prime}\right|(0)+\frac{2}{3} K t^{3}\right)
$$

This gives rise to a uniform upper bound for the length of $\left.\gamma\right|_{\left[0, \tau^{\prime}\right]}$ for $\tau^{\prime} \leq \tau$ measured in $g(0)$. By the completeness of $g(0)$ and basic ODE we conclude that $\gamma$ is defined on $[0, \tau]$.

Proposition 2.5. We have $\bigcup_{\tau} \mathcal{U}(\tau)=T_{p} M$. In other words, the direct limit of $\mathcal{U}(\tau)$ as $\tau \rightarrow 0$ is $T_{p} M$. Indeed, for each $r>0$, there is $\tau>0$ such that $B_{r}(0) \subset \mathcal{U}(\tau)$, where the norm on $T_{p} M$ is induced from $g(0)_{p}$. A similar statement holds true for $\exp _{\bar{p}} \mathcal{L}_{\varepsilon, \tau}$.

Proof. Fix $0<\tau^{*}<\min \{T, 1\}$. Let $r>0$ be given. Let $K$ be an upper bound for $|R i c|$ and $\nabla R$ on $B_{2 r}(p, 0) \times\left[0, \tau^{*}\right]$. Consider $v \in B_{r}(0)$. By $(2.17)$ we have for $\gamma=\gamma_{v}$ (parameterized in $\left.t\right)$

$$
\left|\gamma^{\prime}(t)\right|_{g(0)} \leq e^{K t}\left(r+\frac{2}{3} K t^{3}\right)
$$

as long as $t$ is in the maximal existence interval of $\gamma, t \leq \sqrt{\tau^{*}}$ and $\gamma([0, t]) \subset$ $B_{2 r}(p, 0)$. For such $t$ which also satisfies $t<\sqrt[3]{\frac{r}{2 K}}$ and $t<\frac{1}{K} \ln \frac{9}{8}$ we then have

$$
\left|\gamma^{\prime}\left(t^{\prime}\right)\right|_{g(0)}<\frac{3}{2} r
$$

for all $t^{\prime} \in[0, t]$, whence $\int_{0}^{t}\left|\gamma^{\prime}\right|_{g(0)} d t<\frac{3}{2} r$. Consequently, $\gamma([0, t]) \subset B_{\frac{3}{2} r}(p, 0)$. Since $\gamma(0)=p$, by continuity we then obtain $\gamma([0, t]) \subset B_{\frac{3}{2} r}(p, 0)$ for all $t$ in the maximal existence interval of $\gamma$ such that $0 \leq t \leq t^{*}$, where $t^{*}=$ $\min \left\{\sqrt{\tau^{*}}, \sqrt[3]{\frac{r}{2 K}}, \frac{1}{K} \ln \frac{9}{8}\right\}$. This implies in turn that $\left[0, t^{*}\right]$ is contained in the maximal existence interval of $\gamma$. It follows that $v \in \mathcal{U}(\tau)$ for each $\tau \in\left[0, t^{* 2}\right]$.

Proposition 2.6. For each sufficiently small $\tau>0$, exp $p_{p}^{\mathcal{L}, \tau}$ is a diffeomorphism from a neighborhood of 0 in $T_{p} M$ onto a neighborhood of $p$ in $M$. If $\nabla^{2} R(p, \tau) \geq 0$ for each $\tau$, then this holds for each $\tau$. A similar statement holds for $\exp _{\bar{p}} \mathcal{L}_{\varepsilon, \tau}$.

Proof. First note that $0 \in \mathcal{U}(\tau)$. Indeed, the $\mathcal{L}$-geodesic $\gamma_{0}$ is the constant curve $\gamma_{0} \equiv p$; hence it is defined for all $\tau$. To establish the desired diffeomorphism property, it suffices to show that the differential of $\exp _{p}^{\mathcal{L}, \tau}$ at 0 has zero kernel. For this purpose, consider a nonzero $v \in T_{p} M$ and $\exp _{p}^{\mathcal{L}, \tau}(\theta v)=\gamma_{\theta v}(\tau)$. Obviously, $\left.\operatorname{dexp} p_{p}^{\mathcal{L}, \tau}\right|_{0}(v)=Y_{v}(\sqrt{\tau})$, where $Y_{v}$ (parameterized in $\left.t\right)$ is the $\mathcal{L}$-Jacobi field along $\gamma_{0}$ associated with the family of $\exp _{p}^{\mathcal{L}, \tau}$-geodesics $\gamma_{\theta v}$ (with parameter $\theta$ ). Thus $Y_{v}(0)=0, \nabla_{\frac{d}{d t}} Y_{v}(0)=v$. By $[\mathrm{P} 1,(7.7)]$, the $\mathcal{L}$-Jacobi equation along an $\mathcal{L}$-geodesic $\gamma($ parameterized in $s)$ is

$\nabla_{\frac{d}{d s}} \nabla_{\frac{d}{d s}} Y+\frac{1}{2 s} \nabla_{\frac{d}{d s}} Y+R m(\dot{\gamma}, Y) \dot{\gamma}+2 \nabla_{Y} \operatorname{Ric}(\dot{\gamma}, \cdot)-\nabla_{\dot{\gamma}} \operatorname{Ric}(Y, \cdot)-\frac{1}{2} \nabla^{2} R(Y, \cdot)=0$.

For $\gamma=\gamma_{0}$ this becomes, when parameterized in $t$,

$$
\frac{d^{2} Y}{d t^{2}}-2 t^{2} \nabla^{2} R\left(p, t^{2}\right)(Y, \cdot)=0
$$


Multiplying this equation with $Y$ and integrating, we see that for small $\tau, Y(0)=0$ and $Y(\sqrt{\tau})=0$ imply that $Y \equiv 0$. The same holds for each $\tau$ if $\nabla^{2} R(p, \tau) \geq 0$ for each $\tau$. Applying this to $Y_{v}$ we arrive at the desired conclusions.

Lemma 2.7. A tangent vector $v \in T_{p} M$ is a critical point of $\exp _{p}^{\mathcal{L}, \tau}$ if and only if $q=\exp _{p}^{\mathcal{L}, \tau}(v)$ is conjugate to $p$ along the $\exp _{p}^{\mathcal{L}, \tau}$-geodesic $\gamma_{v}$; i.e. there is a nontrivial $\mathcal{L}$-Jacobi field $J$ along $\gamma_{v}$ with $J(0)=0, J(\tau)=0$.

Proof. This is similar to the corresponding result in the theory of ordinary geodesics in Riemannian geometry. The standard argument there can be applied directly.

Proposition 2.8. If Ric $\geq-c g$ on $[0, \tau]$ for a nonnegative constant $c$, then there exists a minimal $\mathcal{L}_{0, \tau}$-geodesic from $p$ to $q$ for each $q$. Consequently, exp $p_{p}^{\mathcal{L}, \tau}$ is onto.

Proof. Obviously, the $\mathcal{L}$-energy extends to Sobolev curves, i.e. $W^{1,2}$ curves (continuous curves which are in the Sobolev space $W^{1,2}$ in local charts). We work with the reparametrized form $(2.6)$ of the $\mathcal{L}$-energy. Consider a piecewise $C^{1}$ curve $\gamma:[0, \sqrt{\tau}] \rightarrow M$. We have by $(2.12)$,

$$
\int_{0}^{\sqrt{\tau}}\left|\gamma^{\prime}\right|_{g(0)}^{2} \leq 2 e^{2 c \tau}\left(\mathcal{L}(\gamma)+\frac{2 n c}{3} \tau^{\frac{3}{2}}\right)
$$

On the other hand, we have for $0<\tau^{\prime} \leq \tau$,

$$
\begin{aligned}
\mathcal{L}_{0, \tau^{\prime}}\left(\left.\gamma\right|_{\left[0, \sqrt{\tau^{\prime}}\right]}\right) & =\int_{0}^{\sqrt{\tau^{\prime}}}\left(\frac{1}{2}\left|\gamma^{\prime}\right|^{2}+2 R t^{2}\right) d t \leq \int_{0}^{\sqrt{\tau}}\left(\frac{1}{2}\left|\gamma^{\prime}\right|^{2}+2 R t^{2}\right) d t-\int_{\sqrt{\tau^{\prime}}}^{\sqrt{\tau}} 2 R t^{2} d t \\
& \leq \mathcal{L}(\gamma)+\frac{2 n c}{3} \tau^{\frac{3}{2}} .
\end{aligned}
$$

Applying (2.10) we then deduce

$$
d^{2}\left(p, \gamma\left(\sqrt{\tau^{\prime}}\right), 0\right) \leq e^{2 c \tau}\left(\frac{4 n c}{3} \tau^{2}+2 \sqrt{\tau}\left(\mathcal{L}(\gamma)+\frac{2 n c}{3} \tau^{\frac{3}{2}}\right)\right)
$$

For a given $q \in M$ we minimize the $\mathcal{L}$-energy in the reparametrized form $(2.6)$ in the space of Sobolev curves $\gamma:[0, \sqrt{\tau}] \rightarrow M$ which connect $p$ to $q$. Let $\gamma_{k}$ be a minimal sequence. By (2.24), there is a positive number $\rho$ such that all $\gamma_{k}$ are contained in the geodesic ball $B_{\rho}(p, 0)$. By (2.22) we obtain an upper bound for the Dirichlet energy of $\gamma_{k}$ with respect to the metric $g(0)$. It follows that $\gamma_{k}$ subconverges weakly in $W^{1,2}$ to a Sobolev curve $\gamma$. By Sobolev embedding, $\gamma_{k}$ also converges in $C^{0}$. Hence $\gamma$ connects $p$ to $q$. By the obvious lower semicontinuity of the $\mathcal{L}$-energy, we then infer that $\gamma$ is a minimizer of the $\mathcal{L}$-energy in the space of Sobolev curves which connect $p$ to $q$. By standard elliptic regularity, it is a smooth $\mathcal{L}$-geodesic connecting $p$ to $q$.

Set $v=\gamma^{\prime}(0) / 2$. Then $\exp _{p}^{\mathcal{L}, \tau}(v)=q$.

Definition 4. 1) We define the injectivity domain $\Omega(\tau)$ at time $\tau$ to be

$$
\begin{gathered}
\Omega(\tau)=\{q \in M: \text { there is a unique minimal } \mathcal{L} \text {-geodesic } \gamma:[0, \tau] \rightarrow M \\
\text { with } \gamma(0)=p, \gamma(\tau)=q ; q \text { is not conjugate to } p \text { along } \gamma\} .
\end{gathered}
$$

(For the meaning of "conjugate", see Lemma 2.7.)

The cut-locus $\mathcal{C}(\tau)$ is defined to be $M-\Omega(\tau)$.

The corresponding concepts, the $\mathcal{L}_{\varepsilon, \tau}$ injectivity domain $\Omega(\varepsilon, \tau)$ and the cutlocus $\mathcal{C}(\varepsilon, \tau)$ associated with $\mathcal{L}_{\varepsilon, \tau}$-geodesics, are defined in a similar way. 
2) The tangential injectivity domain $\Omega^{T_{p}}(\tau)$ at time $\tau$ is defined to be $\Omega^{T_{p}}(\tau)=\left\{v \in \mathcal{U}(\tau):\left.\gamma_{v}\right|_{[0, \tau]}\right.$ is the unique minimal $\mathcal{L}$-geodesic from $p$ to $\gamma_{v}(\tau) ;$ $\tau$ is not a conjugate time, i.e., $\gamma_{v}(\tau)$ is not conjugate to $p$ along $\left.\gamma_{v}\right\}$.

It is easy to see that $\Omega(\tau)=\exp _{p}^{\mathcal{L}, \tau}\left(\Omega^{T_{p}}(\tau)\right)$.

The tangential $\mathcal{L}_{\varepsilon, \tau}$ injectivity domain $\Omega^{T_{\bar{p}}}(\varepsilon, \tau)$ is defined in a similar way.

Lemma 2.9. $\mathcal{C}(\tau)$ is closed in $M$ for each $\tau \in(0, T)$, and $\bigcup_{0<\tau<T} \mathcal{C}(\tau) \times\{\tau\}$ is closed in $M \times(0, T)$. Consequently, $\Omega(\tau), \Omega^{T_{p}}(\tau), \bigcup_{0<\tau<T} \Omega(\tau) \times\{\tau\}$ and $\bigcup_{0<\tau<T} \Omega^{T_{p}}(\tau) \times\{\tau\}$ are open. exp $p_{p}^{\mathcal{L}, \tau}$ is a smooth diffeomorphism from $\Omega^{T_{p}}(\tau)$ onto $\Omega(\tau)$, depending smoothly on the parameter $\tau . L(q, \tau)$ is a smooth function on $\bigcup_{0<\tau<T} \Omega(\tau) \times\{\tau\}$.

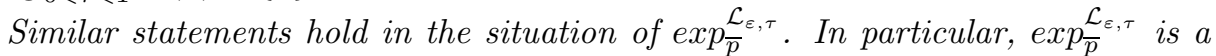
smooth diffeomorphism from $\Omega^{T_{\bar{p}}}(\varepsilon, \tau)$ onto $\Omega(\varepsilon, \tau)$, depending smoothly on $\varepsilon$ and $\tau$, and $L_{\varepsilon, \tau}(q)$ is smooth on $\bigcup_{0<\varepsilon<\tau<T} \Omega(\varepsilon, \tau) \times\{\varepsilon\} \times\{\tau\}$, where $L_{\varepsilon, \tau}(q)$ is defined to be the infimum of $\mathcal{L}_{\varepsilon, \tau}(\gamma)$ for $\gamma:[\varepsilon, \tau] \rightarrow M$ such that $\gamma(\varepsilon)=\bar{p}, \gamma(\tau)=q$.

Proof. All these can easily be established by applying the corresponding standard arguments in the theory of ordinary geodesics in Riemannian geometry.

Lemma 2.10. Let $\gamma$ be a minimal $\mathcal{L}_{0, \tau}$-geodesic. Then $\left.\gamma\right|_{\left[0, \tau^{\prime}\right]}$ is the unique minimal $\mathcal{L}_{0, \tau^{\prime}}$-geodesic from $p$ to $\gamma\left(\tau^{\prime}\right)$ for any $\tau^{\prime} \in(0, \tau)$. Moreover, $\tau^{\prime}$ is not a conjugate time. Thus $\gamma\left(\tau^{\prime}\right) \in \Omega\left(\tau^{\prime}\right)$. We also have $\gamma\left(\tau^{\prime}\right) \in \Omega(\varepsilon, \tau)$ for any $\varepsilon \in(0, \tau)$ and $\tau^{\prime} \in(\varepsilon, \tau)$, where the reference point $\bar{p}$ for $\Omega(\varepsilon, \tau)$ is chosen to be $\gamma(\varepsilon)$.

As a consequence, we have $\Omega^{T_{p}}\left(\tau_{2}\right) \subset \Omega^{T_{p}}\left(\tau_{1}\right)$ for $\tau_{2} \geq \tau_{1}$.

Proof. The arguments in the theory of ordinary geodesics can be applied directly.

Lemma 2.11. Assume that $L(\cdot, \tau)$ is differentiable at $q \in M$. Then there exists at most one minimal $\mathcal{L}_{0, \tau}$-geodesic from $p$ to $q$. Moreover, if $\gamma$ is a minimal $\mathcal{L}_{0, \tau^{-}}$ geodesic from $p$ to $q$, then we have

$$
\nabla L(q, \tau)=2 \sqrt{\tau} \dot{\gamma}(\tau), \quad \nabla l(q, \tau)=\dot{\gamma}(\tau) .
$$

Proof. Consider a point $q$ at which $L(\cdot, \tau)$ is differentiable. Let $\gamma$ be a minimal $\mathcal{L}_{0, \tau}$-geodesic from $p$ to $q$. Given $v \in T_{q} M$, choose a smooth curve $q(\theta), \theta \in(-\epsilon, \epsilon)$ for some $\epsilon>0$ with $q(0)=q, q^{\prime}(0)=v$. Then choose a smooth family of curves $\gamma_{\theta}:[0, \tau] \rightarrow M, \theta \in(-\epsilon, \epsilon)$, such that $\gamma_{0}=\gamma$ and $\gamma_{\theta}(0)=p, \gamma_{\theta}(\tau)=q(\theta)$ for all $\theta$. By the first variation formula $[\mathrm{P} 1,(7.1)]$, it follows that

$$
\left.\frac{d}{d \theta} \mathcal{L}\left(\gamma_{\theta}\right)\right|_{\theta=0}=2 \sqrt{\tau} \dot{\gamma}(\tau) \cdot v
$$

We have $L(q(\theta), \tau) \leq \mathcal{L}\left(\gamma_{\theta}\right)$ for all $\theta$ and $L(q(0), \tau)=\mathcal{L}\left(\gamma_{0}\right)$. Hence the derivative of $L(q(\theta), \tau)$ at $\theta=0$ from the side $\theta>0$ is no larger than the derivative of $\mathcal{L}\left(\gamma_{\theta}\right)$ at $\theta=0$ from the side $\theta>0$, while the derivative of $L(q(\theta), \tau)$ at $\theta=0$ from the side $\theta<0$ is no smaller than the derivative of $\mathcal{L}\left(\gamma_{\theta}\right)$ at $\theta=0$ from the side $\theta<0$. Since $L(\cdot, \tau)$ is differentiable at $q$, this yields

$$
\left.\frac{d}{d \theta} L(q(\theta), \tau)\right|_{\theta=0}=\left.\frac{d}{d \theta} \mathcal{L}\left(\gamma_{\theta}\right)\right|_{\theta=0}=2 \sqrt{\tau} \dot{\gamma}(\tau) \cdot v .
$$

Consequently, the formula (2.25) holds. 
If $\gamma_{1}$ is another minimal $\mathcal{L}_{0, \tau}$-geodesic from $p$ to $q$, then $(2.25)$ implies that $\dot{\gamma}_{1}(\tau)=\dot{\gamma}(\tau)$. It follows that $\gamma_{1}$ coincides with $\gamma$.

Proposition 2.12. Let $\bar{\tau} \in(0, T)$. Assume that Ric $\geq-c g$ on $[0, \bar{\tau}]$ for a nonnegative constant $c$. Then $L(\cdot, \tau)$ is locally Lipschitz and hence almost everywhere differentiable for each $\tau \in(0, \bar{\tau}]$. Moreover, for each compact subset $E$ of $M$, there are positive constants $A_{1}$ and $A_{2}$ such that $\sqrt{\tau} L \leq A_{1}$ on $E \times(0, \bar{\tau}]$ and

$$
|\dot{\gamma}(s)|^{2} \leq \frac{A_{2}}{s}\left(1+\frac{1}{\tau}\right)
$$

for $s \in(0, \tau]$, where $\tau \in(0, \bar{\tau}]$ and $\gamma$ denotes an arbitrary minimal $\mathcal{L}_{0, \tau}$-geodesic from $p$ to $q$ for $q \in E$.

Proof. We first derive an upper bound for $\sqrt{\tau} L(q, \tau)$ on $B_{\rho}(p, \bar{\tau}) \times(0, \bar{\tau}]$ for a given $\rho>0$. By smoothness, there is a positive constant $C$ such that $R \leq C$ on $B_{\rho}(p, \bar{\tau}) \times[0, \bar{\tau}]$. For $q \in B_{\rho}(p, \bar{\tau})$ and $\tau \in(0, \bar{\tau}]$ we choose a minimal geodesic $\gamma:[0, \sqrt{\tau}] \rightarrow B_{\rho}(p, \bar{\tau})$ from $p$ to $q$ with respect to $g(\bar{\tau})$. By $(2.2)$ and (2.6) we have

$$
\mathcal{L}(\gamma) \leq \int_{0}^{\sqrt{\tau}}\left(e^{2 c(\bar{\tau}-t)}\left|\gamma^{\prime}\right|_{g(\bar{\tau})}^{2}+2 C t^{2}\right) d t \leq e^{2 c \bar{\tau}} \frac{d(p, q, \bar{\tau})}{\sqrt{\tau}}+3 C \tau^{\frac{3}{2}} .
$$

It follows that

$$
\sqrt{\tau} L(q, \tau) \leq A(\rho)
$$

where $A(\rho)=e^{2 c \bar{\tau}} \rho+3 C \bar{\tau}^{2}$.

Next consider a given $\rho>0$. Choose $\rho_{1}$ such that $B_{\rho}(p, 0) \subset B_{\rho_{1}}(p, \bar{\tau})$. We set $\rho^{*}=\max \left\{e^{c \bar{\tau}} \sqrt{\frac{4 n c}{3} \bar{\tau}^{2}+2 A\left(\rho_{1}\right)}, 2 \rho\right\}$. By the smoothness of $g$, there is an upper bound $K$ for $|R i c|$ and $|\nabla R|$ on $B_{\rho^{*}}(p, 0) \times[0, \bar{\tau}]$.

Now consider $q_{1}, q_{2} \in B_{\rho}(p, 0)$ and $\tau \in(0, \bar{\tau}]$. Let $\gamma_{i}$ be a minimal $\mathcal{L}_{0, \tau}$-geodesic from $p$ to $q_{i}, i=1,2$. (By Proposition 2.8, they exist.) Let $\gamma_{0}:[0,1] \rightarrow M$ be a minimal geodesic from $q_{1}$ to $q_{2}$ with respect to $g(0)$. By the choice of $\rho^{*}$, the image of $\gamma_{0}$ is obviously contained in $B_{\rho^{*}}(p, 0)$. We claim that the images of $\gamma_{1}$ and $\gamma_{2}$ are also contained in $B_{\rho^{*}}(p, 0)$. Indeed, we have

$$
\int_{0}^{\tau^{\prime}} \sqrt{s}\left(R+\left|\dot{\gamma}_{i}\right|^{2}\right) d s \leq L\left(q_{i}, \tau\right)-\int_{\tau^{\prime}}^{\tau} \sqrt{s} R d s \leq \frac{A\left(\rho_{1}\right)}{\sqrt{\tau}}+\frac{2 n c}{3} \tau^{\frac{3}{2}}
$$

for $i=1,2$ and $\tau^{\prime} \in[0, \tau]$. By (2.10) (applied to $\tau^{\prime}$ ) we then deduce

$$
d\left(p, \gamma_{i}\left(\tau^{\prime}\right), 0\right)^{2} \leq 4 e^{2 c \tau^{\prime}}\left(\frac{1}{2} \sqrt{\tau^{\prime}} L\left(q_{i}, \tau^{\prime}\right)+\frac{n c}{3} \tau^{\prime 2}\right) \leq \rho^{* 2}
$$

for $i=1,2$ and $\tau^{\prime} \in[0, \tau]$. It follows that the images of $\gamma_{1}$ and $\gamma_{2}$ are contained in $B_{\rho^{*}}(p, 0)$.

Next we estimate $\left|\dot{\gamma}_{1}\right|$ and $\left|\dot{\gamma}_{2}\right|$. It is more convenient to handle $\gamma_{1}^{\prime}$ and $\gamma_{2}^{\prime}$. By the arguments in the proof of Lemma 2.4, we deduce

$$
\left|\gamma_{i}^{\prime}\right|\left(t_{2}\right) \geq\left|\gamma_{i}^{\prime}\right|\left(t_{1}\right)-\frac{2}{3} K \bar{\tau}^{3}
$$

for $i=1,2$ and $t_{1}, t_{2} \in[0, \sqrt{\tau}]$, and hence

$$
\left|\gamma_{i}{ }^{\prime}\left(t_{2}\right)\right|^{2} \geq \frac{1}{2}\left|\gamma_{i}{ }^{\prime}\left(t_{1}\right)\right|^{2}-C
$$


for $t_{1}, t_{2} \in[0, \sqrt{\tau}], i=1,2$, where $C=\frac{4}{9} K^{2} \bar{\tau}^{6}$. It follows that

$$
4 s\left|\dot{\gamma}_{i}(s)\right|^{2}=\left|\gamma_{i}^{\prime}\right|^{2} \leq 8\left(\frac{L\left(q_{i}, \tau\right)}{\sqrt{\tau}}+\frac{2 n c}{3} \tau\right)+2 C
$$

for $i=1,2$ and $s \in[0, \tau]$.

To proceed, we set $d=d\left(q_{1}, q_{2}, 0\right)$ and assume that $d<\frac{\tau}{4}$. We define $\hat{\gamma}_{1}(s)=$ $\gamma_{1}(s)$ for $s \in[0, \tau-2 d], \hat{\gamma}_{1}(s)=\gamma_{1}(\tau-2 d+2(s-\tau+2 d))$ for $s \in[\tau-2 d, \tau-d]$ and $\hat{\gamma}_{1}(s)=\gamma_{0}\left(\frac{1}{d}(s-\tau+d)\right)$ for $s \in[\tau-d, \tau]$. Then we have

$$
\begin{aligned}
L\left(q_{2}, \tau\right) \leq & \mathcal{L}\left(\hat{\gamma}_{1}\right) \leq L\left(q_{1}, \tau\right)-\int_{\tau-2 d}^{\tau} \sqrt{s} R\left(\gamma_{1}\right) d s+\int_{\tau-2 d}^{\tau-d} \sqrt{s}\left(R\left(\gamma_{1}\right)+4\left|\dot{\gamma}_{1}\right|^{2}\right) d s \\
& +\int_{\tau-d}^{\tau} \sqrt{s}\left(R\left(\gamma_{0}\right)+\frac{1}{d^{2}}\left|\dot{\gamma}_{0}\right|^{2}\right) d s,
\end{aligned}
$$

where the arguments for $\gamma_{1}$ and $\gamma_{0}$ in the second and third integrals correspond to the defintion of $\hat{\gamma}_{1}$. We have

$$
\begin{aligned}
& -\int_{\tau-2 d}^{\tau} \sqrt{s} R\left(\gamma_{1}\right) d s \leq \frac{2 n c}{3}\left(\tau^{\frac{3}{2}}-(\tau-2 d)^{\frac{3}{2}}\right), \\
& \int_{\tau-2 d}^{\tau-d} \sqrt{s} R\left(\gamma_{1}\right) \leq \frac{2}{3} n K\left((\tau-d)^{\frac{3}{2}}-(\tau-2 d)^{\frac{3}{2}}\right),
\end{aligned}
$$

and

$$
\int_{\tau-d}^{\tau} \sqrt{s} R\left(\gamma_{0}\right) d s \leq \frac{2}{3} n K\left(\tau^{\frac{3}{2}}-(\tau-d)^{\frac{3}{2}}\right) .
$$

By (2.3) we have $\left|\dot{\gamma}_{0}\right|^{2} \leq e^{2 K \tau} d^{2}$; hence

$$
\int_{\tau-d}^{\tau} \sqrt{s} \frac{1}{d^{2}}\left|\dot{\gamma_{0}}\right|^{2} d s \leq \frac{2}{3} e^{2 K \tau}\left(\tau^{\frac{3}{2}}-(\tau-d)^{\frac{3}{2}}\right) .
$$

On the other hand, we infer from (2.33) that

$$
\int_{\tau-2 d}^{\tau-d} 4 \sqrt{s}\left|\dot{\gamma}_{1}\right|^{2} d s \leq 4\left(4 \frac{A\left(\rho_{1}\right)}{\tau}+\frac{8 n c}{3} \tau+C\right)\left((\tau-d)^{\frac{1}{2}}-(\tau-2 d)^{\frac{1}{2}}\right) .
$$

We deduce

$$
L\left(q_{2}, \tau\right) \leq L\left(q_{1}, \tau\right)+I(\tau, d)
$$

where

$$
\begin{aligned}
I(\tau, d) & =\frac{2}{3}\left(2 n c+n K+e^{2 K \tau}\right)\left(\tau^{\frac{3}{2}}-(\tau-2 d)^{\frac{3}{2}}\right) \\
& +4\left(4 \frac{A\left(\rho_{1}\right)}{\tau}+\frac{8 n c}{3} \tau+C\right)\left((\tau-d)^{\frac{1}{2}}-(\tau-2 d)^{\frac{1}{2}}\right) .
\end{aligned}
$$

Similarly, we have

$$
L\left(q_{1}, \tau\right) \leq L\left(q_{2}, \tau\right)+I(\tau, d) .
$$

The desired Lipschitz continuity follows. The estimate (2.28) follows from (2.33).

Finally, we would like to point out that the local Lipschitz continuity of $L(\cdot, \tau)$ also follows from its local semiconcavity, which is given by Proposition 2.15 below. Note however that the proof of Proposition 2.15 below uses some arguments here. 
Proposition 2.13. Assume that the Ricci curvature is bounded from below on $[0, \bar{\tau}]$. Then $L(q, \cdot)$ is locally Lipschitz and hence almost everywhere differentiable on $(0, \bar{\tau}]$ for every $q \in M$. Moreover, $\tau^{\frac{3}{2}}\left|L_{\tau}\right|$ is bounded on $E \times(0, \bar{\tau}]$ for each compact subset $E$ of $M$.

Proof. This is similar to the proof of Proposition 2.12 above. Fix $\rho>0$ and let $\rho^{*}$ and $K$ have the same meanings as in the proof of Proposition 2.12. Consider $q \in B_{\rho}(p, 0)$ and $\tau_{1}, \tau_{2} \in(0, \tau]$ such that $\tau_{1}<\tau_{2}$ and $\tau_{2}<2 \tau_{1}$. Choose a minimal $\mathcal{L}_{0, \tau_{1}}$-geodesic $\gamma_{1}$ from $p$ to $q$ and a minimal $\mathcal{L}_{0, \tau_{2}}$-geodesic $\gamma_{2}$ from $p$ to $q$. As in the proof of Proposition 2.12, the images of $\gamma_{1}$ and $\gamma_{2}$ are contained in $B_{\rho^{*}}(p, 0)$. We define $\hat{\gamma}_{1}(s)=\gamma_{1}(s), s \in\left[0, \tau_{1}\right]$ and $\hat{\gamma}_{1}(s)=q, s \in\left[\tau_{1}, \tau_{2}\right]$. Then

$$
L\left(q, \tau_{2}\right) \leq \mathcal{L}_{0, \tau_{2}}\left(\hat{\gamma}_{1}\right) \leq L\left(q, \tau_{1}\right)+\int_{\tau_{1}}^{\tau_{2}} \sqrt{s} R(q, s) d s \leq L\left(q, \tau_{1}\right)+\frac{2}{3} n K\left(\tau_{2}^{3 / 2}-\tau_{1}^{3 / 2}\right) .
$$

Next we set $\tau_{3}=2 \tau_{1}-\tau_{2}, \hat{\gamma}_{2}(s)=\gamma_{2}(s)$ for $s \in\left[0, \tau_{3}\right]$ and $\hat{\gamma}_{2}(s)=$ $\gamma_{2}\left(\tau_{3}+2\left(s-\tau_{3}\right)\right)$ for $s \in\left[\tau_{3}, \tau_{1}\right]$. Then

$$
\begin{aligned}
L\left(q, \tau_{1}\right) \leq & \mathcal{L}_{0, \tau_{1}}\left(\hat{\gamma}_{2}\right) \leq L\left(q, \tau_{2}\right)-\int_{\tau_{3}}^{\tau_{2}} \sqrt{s} R\left(\gamma_{2}\right) d s \\
& +\int_{\tau_{3}}^{\tau_{1}} \sqrt{s}\left(R\left(\gamma_{2}\right)+4\left|\dot{\gamma}_{2}\right|^{2}\right) d s,
\end{aligned}
$$

where the argument of $\gamma_{2}$ in the last integral on the right hand side is $\tau_{3}+2\left(s-\tau_{3}\right)$. Applying (2.28) we then obtain

$$
L\left(q, \tau_{1}\right) \leq L\left(q, \tau_{2}\right)+\frac{2 n(c+K)}{3}\left(\tau_{1}^{\frac{3}{2}}-\tau_{3}^{\frac{3}{2}}\right)+8 A_{2}\left(1+\frac{1}{\tau}\right)\left(\tau_{1}^{\frac{1}{2}}-\tau_{3}^{\frac{1}{2}}\right) .
$$

Clearly, (2.43) and (2.45) imply the desired Lipschitz continuity and derivative bound.

Proposition 2.14. Assume that the Ricci curvature is bounded from below on $[0, \bar{\tau}]$. Then $L$ is a locally Lipschitz function on $M \times(0, \bar{\tau}]$.

Proof. Combine Proposition 2.12 and Proposition 2.13. To be more precise, we have $\left|L\left(q_{1}, \tau_{1}\right)-L\left(q_{2}, \tau_{2}\right)\right| \leq\left|L\left(q_{1}, \tau_{1}\right)-L\left(q_{1}, \tau_{2}\right)\right|+\left|L\left(q_{1}, \tau_{2}\right)-L\left(q_{2}, \tau_{2}\right)\right|$. We apply the above two propositions to handle the two terms on the right hand side to obtain the desired Lipschitz bound.

Proposition 2.15. Assume that the Ricci curvature is bounded from below on $[0, \bar{\tau}]$. Then $l(\cdot, \tau)$ is locally semi-concave for each $\tau \in(0, \bar{\tau}]$; i.e. for every point $q \in M$ there is a smooth function $\phi$ on a neighborhood $U_{q}$ of $q$ such that $l(\cdot, \tau)+\phi$ is concave in the sense that the composition of $l(\cdot, \tau)+\phi$ with every geodesic in $U_{q}$ is a concave function.

Proof. By [P1, (7.9)], we have for each $\tau \in(0, T), q \in \Omega(\tau)$ and $v \in T_{q} M$,

$$
\operatorname{Hess}_{L}(v, v) \leq \frac{1}{\sqrt{\tau}}|v|^{2}-2 \sqrt{\tau} \operatorname{Ric}(v, v)-\int_{0}^{\tau} \sqrt{s} H(X, Y) d s,
$$


where $X=\dot{\gamma}$ with $\gamma$ denoting the unique minimal $\mathcal{L}$-geodesic from $p$ to $q, Y$ is a suitable extension of $v$ along $\gamma$ such that $|Y(s)|^{2}=\frac{s}{\tau}|v|^{2}$, and

$$
\begin{aligned}
& H(X, Y)=-\nabla_{Y} \nabla_{Y} R-2<\operatorname{Rm}(Y, X) Y, X>-4\left(\nabla_{X} \operatorname{Ric}(Y, Y)-\nabla_{Y} \operatorname{Ric}(Y, X)\right) \\
& \quad-2 \operatorname{Ric}_{\tau}(Y, Y)+2|\operatorname{Ric}(Y, \cdot)|^{2}-\frac{1}{s} \operatorname{Ric}(Y, Y) .
\end{aligned}
$$

To estimate $H(X, Y)$ we fix $\rho>0$ and assume $q \in B_{\rho}(p, 0) \cap \Omega(\tau)$ and $\tau \in(0, \bar{\tau}]$. Let $\rho^{*}$ be given in the proof of Proposition 2.12. As in the proof of Proposition 2.12 , the smoothness of $g$ implies an upper bound $C$ for $\left|\nabla^{2} R\right|,|\nabla R i c|,\left|R i c_{\tau}\right|,|R m|$ and $\mid$ Ric $\mid$ on $B_{\rho^{*}}(p, 0) \times[0, \bar{\tau}]$. By the proof of Proposition 2.12, $\gamma$ is contained in $B_{\rho^{*}}(p, 0)$. Hence we have $|H(X, Y)| \leq \frac{s}{\tau}\left(C\left(3+2 C+\frac{1}{s}\right)+8 C|X|+2 C|X|^{2}\right)|v|^{2}$. Applying (2.28) we then deduce $|H(X, Y)| \leq \frac{C_{1}}{\tau}\left(s+1+\frac{1}{\tau}\right)|v|^{2}$ for a positive constant $C_{1}$. It follows that

$$
\operatorname{Hess}_{L}(v, v) \leq C_{2}|v|^{2}
$$

for a positive constant $C_{2}=C_{2}(\bar{\tau})$. (Note that if the curvature operator is nonnegative, then $H(X, Y)$ can be estimated as in [P1, 7.2].)

We claim that (2.48) holds true for all $q \in B_{\rho}(p, 0)$ in the sense of barriers, provided that $C_{2}$ is chosen large enough. This means that for each point $q \in B_{\rho}(p, 0)$ and each $\varepsilon>0$ we can find a smooth function $f$ on a neighborhood of $q$ (called an $\varepsilon$ barrier at $q)$ such that $f \geq L(\cdot, \tau), f(q)=L(q, \tau)$ and $\operatorname{Hess}_{f}(q)(v, v) \leq\left(C_{2}+\varepsilon\right)|v|^{2}$. Consider $q \in B_{\rho}(p, 0)$. (We can assume that $q \in \mathcal{C}(\tau)$.) Choose a minimal $\mathcal{L}$ geodesic $\gamma$ from $p$ to $q$. For a given $\varepsilon>0$ we define

$$
f=L(\gamma(\varepsilon), \tau)+L_{\varepsilon, \tau}(q),
$$

where $L_{\varepsilon, \tau}$ is defined in Lemma 2.9 with the reference point $\bar{p}=\gamma(\varepsilon)$. By Lemma $2.9, L_{\varepsilon, \tau}$ is smooth at $q$. We can estimate its Hessian at $q$ in the same fashion as above. Indeed, all the relevant lemmas can easily be extended to the situation of $L_{\varepsilon, \tau}$. Then one infers readily that $f$ is an $\varepsilon$-barrier at $q$.

For each $q \in M$ we choose a suitable smooth function on a neighborhood of $q$ (for example $\phi=-C^{\prime} d(q, \cdot, \tau)^{2}$ for a suitable $C^{\prime}$ ) and deduce that

$$
\operatorname{Hess}_{L+\phi} \leq 0
$$

on a neighborhood of $q$ in the sense of barriers. The maximum principle then implies that $L+\phi$ is concave in this neighborhood (see e.g. [Y3]).

Proposition 2.16. Assume that the Ricci curvature is bounded from below on $[0, \tau]$ for $\tau \in(0, T)$. Then the cut-locus $\mathcal{C}(\tau)$ is a closed set of zero measure in $M$. Consequently, $\bigcup_{0<\tau<T} \mathcal{C}(\tau) \times\{\tau\}$ is a closed set of zero measure in $M \times(0, T)$, provided that the Ricci curvature is bounded from below on $[0, \tau]$ for each $\tau \in[0, T)$.

Proof. Set $\mathcal{B}(\tau)=\left\{q \in M: \exists\right.$ more than one minimal $\mathcal{L}_{0, \tau}$-geodesic from $p$ to $\left.q\right\}$ and $\mathcal{D}(\tau)=\left\{q \in M: \exists\right.$ a unique minimal $\mathcal{L}_{0, \tau}$-geodesic $\gamma$ from $p$ to $q ; q$ is conjugate to $p$ along $\gamma\}$. By Proposition 2.8, we have $\mathcal{C}(\tau)=\mathcal{B}(\tau) \cup \mathcal{D}(\tau)$. By Lemma 2.7, $\mathcal{D}(\tau)$ is contained in the set of critical values of $\exp _{p}^{\mathcal{L}, \tau}$. By Sards' theorem, it has zero measure. On the other hand, by Proposition $2.12, L(\cdot, \tau)$ is almost everywhere differentiable. By Lemma 2.11 we then infer that for almost every $q \in M$, there is at most one minimal $\mathcal{L}_{0, \tau}$-geodesic from $p$ to $q$. Hence $\mathcal{B}(\tau)$ 
has zero measure. We conclude that $\mathcal{C}(\tau)$ has zero measure. By Lemma 2.9, it is closed.

Next we assume that the Ricci curvature is bounded below on $[0, \tau]$ for each $\tau$. By Lemma 2.9, $\bigcup_{0<\tau<T} \mathcal{C}(\tau) \times\{\tau\}$ is closed in $M \times(0, T)$ and hence measurable. Then the Fubini theorem implies that it has measure zero.

Instead of using Proposition 2.12 for proving that $\mathcal{B}(\tau)$ has zero measure, we can also use an idea suggested in [KL]. By Sards' theorem, we only need to show that $\mathcal{B}(\tau)^{*}$ has zero measure, where $\mathcal{B}(\tau)^{*}$ is the intersection of $\mathcal{B}(\tau)$ with the set of regular values of $\exp _{p}^{\mathcal{L}, \tau}$. Consider $q \in \mathcal{B}(\tau)^{*}$. Then there are $v_{1}, v_{2} \in T_{p} M$ such that $v_{1} \neq v_{2}, \exp _{p}^{\mathcal{L}, \tau}\left(v_{1}\right)=\exp _{p}^{\mathcal{L}, \tau}\left(v_{2}\right)=q$, and $L\left(v_{1}, \tau\right)=L\left(v_{2}, \tau\right)$, where $L(v, \tau)=\mathcal{L}\left(\gamma_{v}\right)$. Since $v_{1}$ and $v_{2}$ are noncritical for $\exp _{p}^{\mathcal{L}, \tau}$, there are disjoint neighborhoods $U_{1}$ of $v_{1}$ and $U_{2}$ of $v_{2}$ such that $F_{1}=\left.\exp _{p}^{\mathcal{L}, \tau}\right|_{U_{1}}$ and $F_{2}=\left.\exp _{p}^{\mathcal{L}, \tau}\right|_{U_{2}}$ are diffeomorphisms onto their common image $U$, which is a neighborhood of $q$.

To proceed, we define $L_{*}(v, w)=L(v, \tau)-L(w, \tau)$, and set $S=\{(v, w) \in$ $\left.U_{1} \times U_{2}: F_{1}(v)=F_{2}(w)\right\}$. Obviously, $S$ is an $n$-dimensional submanifold of $U_{1} \times U_{2}$. We claim that 0 is a regular value of $\left.L_{*}\right|_{S}$. Indeed, consider a curve $(v(t), w(t))$ in $S$ which represents a tangent vector $\left(v^{\prime}(0), w^{\prime}(0)\right)$ of $S$ at a given point $(v(0), w(0))$. Since $\exp _{p}^{\mathcal{L}, \tau}(v(t))=\exp p_{p}^{\mathcal{L}, \tau}(w(t))$, we have

$$
d\left(\exp _{p}^{\mathcal{L}, \tau}\right)_{v(0)}\left(v^{\prime}(0)\right)=d\left(\exp _{p}^{\mathcal{L}, \tau}\right)_{w(0)}\left(w^{\prime}(0)\right) .
$$

On the other hand, by the first variation formula $[(7.1), \mathrm{P}]$ for the $\mathcal{L}$ energy, we have

$$
\frac{d L^{*}(v(t), w(t))}{d t}(0)=2 \sqrt{\tau}\left(<\dot{\gamma}_{v(0)}(0), Y_{1}>-<\dot{\gamma}_{w(0)}(0), Y_{2}>\right)
$$

where $Y_{1}=d\left(\exp _{p}^{\mathcal{L}, \tau}\right)_{v(0)}\left(v^{\prime}(0)\right)$ and $Y_{2}=d\left(\exp _{p}^{\mathcal{L}, \tau}\right)_{w(0)}\left(w^{\prime}(0)\right)$. Since $v(0) \neq w(0)$, we have $\dot{\gamma}_{v(0)}(\tau) \neq \dot{\gamma}_{w(0)}(\tau)$. It follows that $d L_{((v(0), w(0))}^{*}\left(\left(v^{\prime}(0), w^{\prime}(0)\right)\right) \neq 0$. By the implicit function theorem, $\left.L_{*}\right|_{S} ^{-1}(0)$ is an $(n-1)$-dimensional submanifold of $U_{1} \times U_{2}$. Consequently, $S^{*}=\pi_{1} \circ F\left(\left.L^{*}\right|_{S} ^{-1}(0)\right)$ is an $(n-1)$-dimensional submanifold, where $F=\left(F_{1}, F_{2}\right)$ and $\pi_{1}$ denotes the projection from $U \times U$ to the first factor. We call $S^{*}$ a local container for $\mathcal{B}(\tau)^{*}$.

It is easy to see that $\mathcal{B}(\tau)^{*}$ is contained in a countable union of local containers. Hence it has zero measure.

Lemma 2.17. Assume that the Ricci curvature is bounded from below on $[0, \tau]$ for each $0<\tau<T$. Then $\nabla l$ and $l_{\tau}$ exist almost everywhere and are measurable on $M \times(0, T)$.

Proof. This follows from Lemma 2.9 and Proposition 2.16, or from Proposition 2.14 .

Theorem 2.18. Assume that the curvature operator is nonnegative for each $\tau \in$ $[0, T)$. For each $\bar{\tau} \in(0, T)$, there is a positive constant depending only on the dimension $n$ and the magnitude of $\frac{\bar{\tau}}{T-\bar{\tau}}$ such that

$$
R \leq \frac{C l}{\tau}
$$

everywhere on $M \times(0, \bar{\tau}]$,

$$
|\nabla l|^{2} \leq \frac{C l}{\tau}
$$


almost everywhere in $M$ for each $\tau \in(0, \bar{\tau}]$,

$$
\left|\sqrt{l}\left(q_{1}, \tau\right)-\sqrt{l}\left(q_{2}, \tau\right)\right| \leq \sqrt{\frac{C}{4 \tau}} d\left(q_{1}, q_{2}, \tau\right)
$$

for all $\tau \in(0, \bar{\tau}]$ and all $q_{1}, q_{2} \in M$, and

$$
\left|l_{\tau}\right| \leq \frac{C l}{\tau}
$$

almost everywhere in $(0, \bar{\tau}]$ for each $q \in M$. (Note that $\frac{\bar{\tau}}{T-\bar{\tau}}$ is understood to be zero when $T=\infty$. Thus $C$ depends only on $n$ in this case.) Moreover, we have the following Harnack inequality:

$$
\left(\frac{\tau_{1}}{\tau_{2}}\right)^{C} \leq \frac{l\left(q, \tau_{2}\right)}{l\left(q, \tau_{1}\right)} \leq\left(\frac{\tau_{2}}{\tau_{1}}\right)^{C}
$$

for all $q \in M$ and $\tau_{1}, \tau_{2} \in(0, \bar{\tau}]$ with $\tau_{2}>\tau_{1}$.

Proof. By $[\mathrm{P} 1,(7.16)]$, we have for each $\bar{\tau} \in(0, T)$,

$$
|\nabla l|^{2}+R \leq C \frac{l}{\tau}
$$

on $\bigcup_{0<\tau \leq \bar{\tau}} \Omega(\tau) \times\{\tau\}$ for a positive constant $C$ depending only on the dimension $n$ and the magnitude of $\frac{\bar{\tau}}{T-\bar{\tau}}$. The estimates (2.53) and (2.54) follow from this, Proposition 2.12, and Proposition 2.16. Now the estimate (2.54) can be rewritten as

$$
|\nabla \sqrt{l}|^{2} \leq \frac{C}{4 \tau}
$$

which implies (and is equivalent to) (2.55). Indeed, given $\tau \in(0, \bar{\tau}]$ and $q \in M$, we can apply (2.59) to derive (2.55) along almost every radial geodesic (for the metric $g(\tau))$ starting at $q$. By continuity, it holds along every radial geodesic. Hence $(2.55)$ holds for all $q_{1}, q_{2} \in M$.

Next we derive (2.56). Fix $q \in M$. By Proposition 2.13, $l_{\tau}(q, \tau)$ exists for almost every $\tau$. Consider $\tau \in(0, \bar{\tau}]$ such that $l_{\tau}(q, \tau)$ exists. Observe that $(2.56)$ is invariant under the rescaling $g(\tau) \rightarrow \frac{1}{a} g(a \tau)$; hence it suffices to prove it in the case that $\bar{\tau}>1$ and $\tau=1$. We consider $\tau_{1}=1, \tau_{2} \in(1, \bar{\tau}]$ with $\tau_{2}<2$ and the curves $\gamma_{1}, \gamma_{2}$ and $\hat{\gamma}_{2}$ as in the proof of Proposition 2.13. By Hamilton's Harnack inequality [P1, (11.1)], $R_{\tau} \leq 0$ and hence $R(q, s) \leq R(q, 1)$ for $s \geq 1$. By (2.53) we then infer as in (2.43),

$$
L\left(q, \tau_{2}\right) \leq L(q, 1)+C L(q, 1)\left(\tau_{2}^{\frac{3}{2}}-1\right) .
$$

On the other hand, by Lemma 2.10 we have $\gamma_{2}(s) \in \Omega(s)$ for $s \in\left(0, \tau_{2}\right)$. Hence we can apply (2.58) to (2.44) to deduce

$$
L(q, 1) \leq L\left(q, \tau_{2}\right)+C L\left(q, \tau_{2}\right)\left(\sqrt{\tau_{2}}-\sqrt{2-\tau_{2}}\right) .
$$

Obviously, (2.60) and (2.61) imply $\left|l_{\tau}(q, 1)\right| \leq C l(q, 1)$ for a positive constant $C$ depending only on $n$.

Integrating (2.56) yields the Harnack estimate (2.57).

Similar estimates for $l$ hold in the case of bounded sectional curvature. 
Proposition 2.19. Assume that the sectional curvature is bounded on $[0, \bar{\tau}]$. Then there is a positive constant $C=C\left(\tau^{*}\right)$ for every $\tau^{*} \in(0, \bar{\tau})$ with the following properties. For each $\tau \in\left(0, \tau^{*}\right]$ we have

$$
|\nabla l|^{2} \leq \frac{C}{\tau}(l+\tau+1)
$$

almost everywhere in $M$. For each $q \in M$ we have

$$
\left|l_{\tau}\right| \leq \frac{C}{\tau}(l+\tau+1)
$$

almost everywhere in $\left(0, \tau^{*}\right]$.

Proof. Consider $\tau^{*} \in(0, \bar{\tau}), \tau \in\left(0, \tau^{*}\right]$ and $q \in M$ at which $l(\cdot, \tau)$ is differentiable. By the assumption and the local interior estimates in $[\mathrm{S}]$, we have global bounds for $|R m|$ and $|\nabla R|$ on $[0, \tau]^{*}$. By the arguments in the proof of Proposition 2.12 we then deduce, for a minimal $\mathcal{L}_{0, \tau}$-geodesic $\gamma$ from $p$ to $q$,

$$
|\dot{\gamma}|^{2} \leq \frac{C}{s}(l(q, \tau)+\tau+1)
$$

for a positive constant $C=C\left(\tau^{*}\right)$. Taking $s=\tau$ in (2.64) and applying Proposition 2.12 and Lemma 2.11 we then arrive at (2.62).

The estimate (2.63) follows from (2.64) and the arguments in the proof of Proposition 2.13 .

Lemma 2.20. Let $A$ be a compact set of zero measure in a Riemannian manifold $(N, h)$ of dimension $n$, where $h$ denotes the metric. Let $U_{r}(A)$ denote the open $r$ tubular neighborhood of $A$, i.e. $U_{r}(A)=\{q \in N: d(A, q)<r\}$. Then $\operatorname{vol}\left(U_{r}(A)\right) \rightarrow$ 0 as $r \rightarrow 0$.

Proof. The Lebesgue measure equals the $n$-dimensional spherical measure; see [F, 2.10.35] (the proof there for the Euclidean spaces easily extends to general Riemannian manifolds). Hence the $n$-dimensional spherical measure of $A$ is zero. Hence, we have for each $r>0$, a countable collection of geodesic balls $B_{\rho(r, i)}(q(r, i))$ with positive radii $\rho(r, i)$ not exceeding $r$, such that $A \subset \bigcup_{i} B_{\rho(r, i)}(q(r, i))$ and $\sum_{i} \operatorname{vol}\left(B_{\rho(r, i)}(q(r, i))\right) \rightarrow 0$ as $r \rightarrow 0$. Since $A$ is compact, we can find for each $r>0$ finitely many $B_{\rho(r, i)}(q(r, i))$ to cover $A$. Passing to those finite coverings, we can assume from the beginning that for each $r$ the collection $\left\{B_{\rho(r, i)}(q(r, i))\right\}$ is finite. We set $\rho(r)=\min _{i} \rho(r, i)$. Then $U_{\rho(r)}(A) \subset \bigcup_{i} B_{2 \rho(r)}(q(r, i))$. Obviously, $\sum_{i} \operatorname{vol}\left(B_{2 \rho(r)}(q(r, i))\right) \rightarrow 0$ as $r \rightarrow 0$. It follows that $\operatorname{vol}\left(U_{r}(A)\right) \rightarrow 0$ as $r \rightarrow 0$. (Instead of the $n$-dimensional spherical measure, one can also use the $n$-dimensional Hausdorff measure, which also equals the Lebesgue measure.)

Lemma 2.21. Assume that the Ricci curvature is bounded from below on $[0, \bar{\tau}]$. Then for every $\tau \in(0, \bar{\tau}]$, it follows that

$$
\int_{M} l \Delta \phi d q \leq \int_{* M} \phi \Delta l d q
$$

for nonnegative smooth functions $\phi$ with compact support, where the integral $\int_{* M}$ means $\liminf _{\epsilon \rightarrow 0} \int_{M-U_{\epsilon}}$, with $U_{\epsilon}=U_{\epsilon}(\mathcal{C}(\tau))$ denoting the open $\epsilon$-tubular neighbor- 
hood of $\mathcal{C}(\tau)$ with respect to $g(\tau)$. Consequently, we have

$$
-\int_{M} \nabla l \cdot \nabla \phi d q \leq \int_{* M} \phi \Delta l d q
$$

for nonnegative Lipschitz functions $\phi$ with compact support.

Proof. Consider $q_{0} \in M$. By Proposition 2.15, there is a neighborhood $U$ of $q_{0}$ and a smooth function $\psi$ on $U$ such that $l+\psi$ is concave. We can assume that $l+\psi$ is actually strictly concave; i.e. it is the sum of a concave function and a smooth concave function with negative Hessian. By [GW] or [Y3], there exists a sequence of smooth concave functions $f_{k}$ with negative Hessian on a neighborhood $\hat{U} \subset U$ of $q_{0}$ such that: 1) the $f_{k}$ converge uniformly to $l+\psi$ on $\hat{U}$, and 2) the derivatives of $f_{k}$ converge uniformly to the derivatives of $l+\psi$ on $\hat{U}-U_{\epsilon}$ for each $\epsilon>0$.

Let $\phi$ be a nonnegative smooth function with compact support contained in $\hat{U}$. We have

$$
\int_{M} f_{k} \Delta \phi d q=\int_{M} \Delta f_{k} \phi d q=\int_{M-U_{\epsilon}} \Delta f_{k} \phi d q+\int_{U_{\epsilon}} \Delta f_{k} \phi d q \leq \int_{M-U_{\epsilon}} \Delta f_{k} \phi d q .
$$

Taking the limit we deduce

$$
\int_{M}(l+\psi) \Delta \phi d q \leq \int_{M-U_{\epsilon}} \Delta(l+\psi) \phi d q
$$

It follows that

$$
\int_{M}(l+\psi) \Delta \phi d q \leq \liminf _{\epsilon \rightarrow 0} \int_{M-U_{\epsilon}} \Delta(l+\psi) \phi d q .
$$

Since $\mathcal{C}(\tau)$ is closed and has zero measure and the support of $\phi$ is compact, we can apply Lemma 2.20 to deduce

$$
\lim _{\epsilon \rightarrow 0} \int_{M-U_{\epsilon}} \Delta \psi \phi d q=\int_{M} \Delta \psi \phi d q=\int_{M} \psi \Delta \phi d q
$$

Hence we conclude that

$$
\int_{M} l \Delta \phi d q \leq \liminf _{\epsilon \rightarrow 0} \int_{M-U_{\epsilon}} \phi \Delta l d q .
$$

Since $q_{0}$ is arbitrary, we deduce by using a partition of unity that (2.65) holds true for all nonnegative smooth functions $\phi$ with compact support.

Lemma 2.22. We have on $\bigcup_{\tau} \Omega(\tau) \times\{\tau\}$,

$$
\begin{aligned}
l_{\tau}-\frac{R}{2}+\frac{|\nabla l|^{2}}{2}+\frac{l}{2 \tau} & =0, \\
l_{\tau}-\Delta l+|\nabla l|^{2}-R+\frac{n}{2 \tau} & \geq 0,
\end{aligned}
$$

and

$$
\Delta l-\frac{|\nabla l|^{2}}{2}+\frac{R}{2}+\frac{l-n}{2 \tau} \leq 0 .
$$

Moreover, (2.73) becomes an equality at a point if and only if (2.74) becomes an equality at that point. 
Proof. The equation (2.72) follows from $[\mathrm{P} 1,(7.5)]$ and $[\mathrm{P} 1,(7.6)]$. The inequality (2.73) is $[\mathrm{P} 1,(7.13)]$, while the inequality $(2.74)$ is $[\mathrm{P} 1,(7.14)]$. On the other hand, the left hand side of (2.73) equals the left hand side of (2.72) minus the left hand side of (2.74). The statement about the equality cases follows.

Theorem 2.23. Assume that the Ricci curvature is bounded from below on $[0, \tau]$ for each $\tau \in(0, T)$. Then the differential inequality (2.73) holds true on $M \times(0, T)$, when $\Delta l$ is interpreted in the weak sense. Namely we have

$$
\int_{\tau_{1}}^{\tau_{2}} \int_{M}\left\{\nabla l \cdot \nabla \phi+\left(l_{\tau}+|\nabla l|^{2}-R+\frac{n}{2 \tau}\right) \phi\right\} d q d \tau \geq 0
$$

for all $0<\tau_{1}<\tau_{2}<T$ and nonnegative Lipschitz functions $\phi$ on $M \times\left[\tau_{1}, \tau_{2}\right]$ with compact support. Similarly, the differential inequality (2.74) holds true for each $\tau \in(0, T)$ in the weak sense; i.e.

$$
\int_{M}\left\{-\nabla l \cdot \nabla \phi+\phi\left(-\frac{|\nabla l|^{2}}{2}+\frac{R}{2}+\frac{l-n}{2 \tau}\right)\right\} d q \leq 0
$$

for all nonnegative Lipschitz functions $\phi$ on $M$ with compact support.

Proof. We first prove (2.75). Let $\phi$ be a nonnegative Lipschitz function on $M \times$ $\left[\tau_{1}, \tau_{2}\right]$ with compact support. By Proposition 2.12, Proposition 2.13, Lemma 2.17 and Lemma 2.21 we have

$$
\begin{gathered}
\int_{\tau_{1}}^{\tau_{2}} \int_{M}\left\{\nabla l \cdot \nabla \phi+\left(l_{\tau}+|\nabla l|^{2}-R+\frac{n}{2 \tau}\right) \phi\right\} d q d \tau \\
\geq \int_{\tau^{1}}^{\tau_{2}} \int_{M}^{*}\left(l_{\tau}-\Delta l+|\nabla l|^{2}-R+\frac{n}{2 \tau}\right) \phi d q d \tau
\end{gathered}
$$

where the integral $\int_{M}^{*}$ means the limsup of the integral on $M-U_{\epsilon}$ as $\epsilon \rightarrow 0$. By the differential inequality (2.73) in Lemma 2.22, the right hand side in (2.77) is nonnegative.

The inequality (2.76) follows from a similar argument.

\section{BASIC PROPERTIES OF THE $l$-FUNCTION II}

Lemma 3.1. Assume that the Ricci curvature is bounded from below on $[0, \tau]$. Then the minimum of $l(\cdot, \tau)$ does not exceed $\frac{n}{2}$.

Proof. We have the differential inequality $[\mathrm{P} 1,(7.10)]$ on $\bigcup \Omega(\tau) \times\{\tau\}$,

$$
\Delta L \leq-2 \sqrt{\tau} R+\frac{1}{\sqrt{\tau}}-\frac{1}{K}
$$

which is obtained by taking the trace in (2.46). Here $K$ is defined on page 16 in $[\mathrm{P} 1]$. Combining this with the equation $[\mathrm{P} 1,(7.5)]$,

$$
L_{\tau}=2 \sqrt{\tau} R-\frac{1}{2 \tau} L+\frac{1}{\tau} K
$$

yields the differential inequality $[\mathrm{P} 1,(7.15)]$ :

$$
\bar{L}_{\tau}+\Delta \bar{L} \leq 2 n,
$$

where $\bar{L}(q, \tau)=2 \sqrt{\tau} L(q, \tau)$. By the argument in the proof of Proposition 2.15, one readily shows that under the Ricci curvature assumption (3.3) holds true in the sense of barriers. More precisely, for each $q \in M$, each $\tau \in(0, T)$ and each $\varepsilon>0$, there is a smooth function $u$ (an $\varepsilon$-barrier at $(q, \tau))$ on a neighborhood of $(q, \tau)$ in 
$M \times[\tau, T)$ such that $u \geq \bar{L}, u(q, \tau)=\bar{L}(q, \tau)$ and $u_{\tau}(q, \tau)+\Delta u(q, \tau) \leq 2 n+\varepsilon$. (We use the forward interval $[\tau, T)$ here because the left hand side of $(3.3)$ is the backward heat operator.) By Lemma 2.3 the minimum of $l(\cdot, \tau)$ and hence of $\bar{L}(\cdot, \tau)$ is achieved for every $\tau$. Consequently, the maximum principle implies that the minimum of $\bar{L}(\cdot, \tau)-2 n \tau$ is nonincreasing. The desired bound for the minimum of $l$ follows.

The details of the said maximum principle are as follows. Set $v=\bar{L}-2 n \tau$. Then $v$ satisfies $v_{\tau}+\Delta v \leq 0$ in the sense of barriers. Let $h(\tau)=\min v(\cdot, \tau)$. Consider $\tau$ and a minimum point $q$ for $v(\cdot, \tau)$. For $\varepsilon>0$ let $u$ be an $\varepsilon$-barrier of $v$ at $(q, \tau)$. Then we have for $\tau^{\prime}>\tau$ sufficiently close to $\tau$,

$$
\frac{h\left(\tau^{\prime}\right)-h(\tau)}{\tau^{\prime}-\tau} \leq \frac{v\left(q, \tau^{\prime}\right)-v(q, \tau)}{\tau^{\prime}-\tau} \leq \frac{u\left(q, \tau^{\prime}\right)-u(q, \tau)}{\tau^{\prime}-\tau} .
$$

Taking the limit we obtain

$$
\frac{d^{+} h}{d \tau} \leq \frac{\partial u}{\partial \tau}(q, \tau) \leq-\Delta u(q, \tau)+\varepsilon
$$

where $\frac{d^{+} h}{d \tau}=\lim \sup _{\tau^{\prime} \rightarrow 0^{+}} \frac{h\left(\tau^{\prime}\right)-h(\tau)}{\tau^{\prime}-\tau}$. Obviously, $q$ is a minimum point for $u(\cdot, \tau)$, whence $\Delta u(q, \tau) \geq 0$. Letting $\varepsilon \rightarrow 0$ we then arrive at

$$
\frac{d^{+} h}{d \tau} \leq 0
$$

Consequently, $h$ is nonincreasing; cf. [H].

Next we present a lower bound and an upper bound for $l$ at any given time in terms of the distance at the same time, which can be compared with Lemma 2.3. The basic idea of the lower bound was communicated to us by Perelman. To work out the precise dependence of the estimate on $\tau$, we formulate it in a scaling invariant form.

Lemma 3.2. Assume that the curvature operator is nonnegative on $(0, T)$. Let $\bar{\tau} \in(0, T)$. Then we have on $(0, \bar{\tau}]$,

$$
-l(x, \tau)-1+C_{1} \frac{d^{2}(x, q, \tau)}{\tau} \leq l(q, \tau) \leq 2 l(x, \tau)+C_{2} \frac{d^{2}(x, q, \tau)}{\tau}
$$

for all $x, q \in M$, where $C_{1}$ and $C_{2}$ are positive constants depending only on the dimension $n$ and the magnitude of $\frac{\bar{\tau}}{T-\bar{\tau}}$. (In particular, $C_{1}$ and $C_{2}$ depend only on $n$ if $T=\infty$.)

Proof. It follows from the Lipschitz estimate (2.55) in Theorem 2.18 that

$$
\sqrt{l}(q, \tau) \leq \sqrt{l}(x, \tau)+\sqrt{\frac{C}{4 \tau}} d(x, q, \tau) .
$$

Squaring it we arrive at the upper bound in (3.7). Next we derive the lower bound. Note that $l$ and the quantity $d^{2}(x, q, \tau) / \tau$ are both invariant under the rescaling $g(\tau) \rightarrow a^{-1} g(a \tau)$. Hence it suffices to prove (3.7) for the case $\tau=1$. 
Since $\Omega(1)$ is dense in $M$, it suffices to consider the case $x, q \in \Omega(1)$. Let $\gamma_{x}, \gamma_{q}$ be the minimal $\mathcal{L}_{0,1}$-geodesics from $p$ to $x, q$ respectively. Then

$$
\begin{aligned}
d(x, q, 1) & =\int_{0}^{1} \frac{d}{d s} d\left(\gamma_{x}(s), \gamma_{q}(s), s\right) d s \\
& =\int_{0}^{1}\left[\frac{\partial d}{\partial s}\left(\gamma_{x}(s), \gamma_{q}(s), s\right)+\nabla_{I} d \cdot \dot{\gamma}_{x}(s)+\nabla_{I I} d \cdot \dot{\gamma}_{q}(s)\right] d s
\end{aligned}
$$

where $\nabla_{I}$ refers to the gradient with respect to the first argument, and $\nabla_{I I}$ that with respect to the second argument.

By Lemma 2.10 and Lemma 2.11 we have $\dot{\gamma}_{x}(s)=\nabla l\left(\gamma_{x}(s), s\right)$ and $\dot{\gamma}_{q}(s)=$ $\nabla l\left(\gamma_{q}(s), s\right)$ for all $s \in(0,1]$. Since the scalar curvature is nonnegative, it follows that

$$
l\left(\gamma_{q}(s), s\right)=\frac{1}{2 \sqrt{s}} \mathcal{L}_{0, s}\left(\left.\gamma_{q}\right|_{[0, s]}\right) \leq \frac{1}{2 \sqrt{s}} \mathcal{L}_{0,1}\left(\gamma_{q}\right)=\frac{1}{\sqrt{s}} l(q, 1) .
$$

Similarly, we have

$$
l\left(\gamma_{x}(s), s\right) \leq \frac{1}{\sqrt{s}} l(x, 1) .
$$

Hence we can apply (2.54) to deduce

$$
\left|\dot{\gamma_{q}}(s)\right| \leq \sqrt{C} s^{-3 / 4} \sqrt{l}(q, 1), \quad\left|\dot{\gamma_{x}}(s)\right| \leq \sqrt{C} s^{-3 / 4} \sqrt{l}(x, 1) .
$$

Next we estimate $\frac{\partial}{\partial s} d\left(\gamma_{x}(s), \gamma_{q}(s), s\right)$. Set $r_{1}(s)=s^{3 / 4}(l(q, 1)+1)^{-1 / 2}$. By $(3.8)$ and (3.10) we have for $\bar{q}$ with $d\left(\bar{q}, \gamma_{q}(s), s\right) \leq r_{1}(s)$,

$$
\sqrt{l}(\bar{q}, s) \leq \sqrt{l}\left(\gamma_{q}(s), s\right)+\frac{\sqrt{C}}{2 \sqrt{s}} r_{1}(s) \leq\left(s^{-1 / 4}+\frac{\sqrt{C}}{2}\right) \sqrt{l(q, 1)+1} .
$$

By (2.53) we then infer

$$
R(\bar{q}, s) \leq C s^{-1}\left(s^{-1 / 4}+\frac{\sqrt{C}}{2}\right)^{2}(l(q, 1)+1) .
$$

Similarly, we have

$$
R(\bar{q}, s) \leq C s^{-1}\left(s^{-1 / 4}+\frac{\sqrt{C}}{2}\right)^{2}(l(x, 1)+1)
$$

for $\bar{q}$ with $d\left(\bar{q}, \gamma_{x}(s), s\right) \leq r_{2}(s)$, where $r_{2}(s)=s^{3 / 4}(l(x, 1)+1)^{-1 / 2}$.

We set $r_{0}(s)=s^{3 / 4}(l(q, 1)+l(x, 1)+1)^{-1 / 2}$. Applying $[\mathrm{P} 1,(8.3(\mathrm{~b}))]$ to the present situation of the backward Ricci flow, we obtain

$$
\frac{\partial}{\partial s} d\left(\gamma_{x}(s), \gamma_{q}(s), s\right) \leq 2(n-1)\left(\frac{2}{3} K r_{0}(s)+r_{0}(s)^{-1}\right),
$$

where $(n-1) K$ is an upper bound for the Ricci curvature at time $s$ on the geodesic balls $d\left(\gamma_{q}(s), \cdot, s\right) \leq r_{0}(s)$ and $d\left(\gamma_{x}(s), \cdot, s\right) \leq r_{0}(s)$. By the estimates (3.14) and (3.15) and the nonnegativity of the Ricci curvature, we can choose

$$
K=\frac{C}{n-1} s^{-1}\left(s^{-1 / 4}+\frac{\sqrt{C}}{2}\right)^{2}(l(q, 1)+l(x, 1)+1) .
$$


Hence we deduce

$$
\begin{aligned}
\frac{\partial}{\partial s} d\left(\gamma_{x}(s), \gamma_{q}(s), s\right) \leq & \frac{4 C}{3}\left(\left(1+\frac{3(n-1)}{2 C}\right) s^{-3 / 4}+\sqrt{C} s^{-1 / 2}+\frac{C}{4} s^{-1 / 4}\right) . \\
& \sqrt{l(q, 1)+l(x, 1)+1} .
\end{aligned}
$$

Combining (3.9), (3.12) and (3.18) we arrive at

$$
\begin{aligned}
d(x, q, 1) \leq & 4 \sqrt{C} \sqrt{l}(q, 1)+4 \sqrt{C} \sqrt{l}(x, 1)+ \\
& \frac{4 C}{3}\left(4\left(1+\frac{3(n-1)}{2 C}\right)+2 \sqrt{C}+\frac{C}{3}\right) \sqrt{l(q, 1)+l(x, 1)+1} \\
\leq & \left(17 C^{2}+8(n-1)\right) \sqrt{l(q, 1)+l(x, 1)+1} .
\end{aligned}
$$

(We may assume that $C \geq 1$.) This estimate yields the lower bound in (3.7) for $\tau=1$.

Theorem 3.3. Assume either that the curvature operator is nonnegative for each $\tau$ or that the sectional curvature is bounded on $[0, \tau]$ for each $\tau$. Then the inequality (2.75) holds true for all $0<\tau_{1}<\tau_{2}$ and nonnegative locally Lipschitz functions $\phi$ on $M \times\left[\tau_{1}, \tau_{2}\right]$ such that $\phi \leq \bar{C} e^{-\bar{c} l}$ and $|\nabla \phi| \leq \bar{C} e^{-\overline{c l}}$ for positive constants $\bar{C}$ and $\bar{c}$ depending on $\phi$ and the magnitude of $\tau_{1}^{-1}$. Similarly, the inequality (2.76) holds true for nonnegative locally Lipschitz functions $\phi$ on $M$ such that $\phi \leq \bar{C} e^{-\overline{c l}}$ and $|\nabla \phi| \leq \bar{C} e^{-\bar{c} l}$ with positive constants $\bar{C}$ and $\bar{c}$ depending on $\phi$ and the magnitude of $\tau^{-1}$. In both cases, the involved integrals are absolutely convergent. In particular, we obtain by choosing $\phi=\tau^{-\frac{n}{2}} e^{-l}$ in (2.75),

$$
\int_{\tau_{1}}^{\tau_{2}} \int_{M}\left(l_{\tau}-R+\frac{n}{2 \tau}\right) e^{-l} \tau^{-\frac{n}{2}} d q d \tau \geq 0
$$

Proof. We present the case of (2.75), while the case of (2.76) is similar and easier. We can assume that $M$ is noncompact.

Let $0<\tau_{1}<\tau_{2}$ and $\phi$ as specified in the statements of the theorem be given.

Part 1. Absolute Convergence

We first show that the integral on the left hand side of (2.75) converges absolutely. Indeed, we can take the absolute value of every term in the integrand and still have convergence. In the case of bounded sectional curvature we apply Proposition 2.19 to deduce for each $\tau$,

$$
\int_{\tau_{1}}^{\tau_{2}} \int_{M}\left(|\nabla l| \cdot|\nabla \phi|+\left(\left|l_{\tau}\right|+|\nabla l|^{2}+|R|+\frac{n}{2 \tau}\right)|\phi|\right) d q d \tau \leq \int_{\tau_{1}}^{\tau_{2}} \int_{M} \tilde{C} e^{-\frac{\bar{c}}{2} l} d q d \tau
$$

for a suitable positive constant $\tilde{C}$ depending on $\phi$ and the magnitude of $\tau_{1}^{-1}$. Lemma 2.3 yields

$$
l(q, \tau) \geq \frac{e^{-2 c \tau_{2}}}{4 \tau} d^{2}\left(p, q, \tau_{1}\right)-\frac{n c}{3} \tau_{2}
$$

for $\tau \in\left[\tau_{1}, \tau_{2}\right]$ and a postive constant $c$ (a lower bound for the Ricci curvature). By Lemma 2.1, we have $d(p, q, \tau) \leq e^{C\left(\tau_{2}-\tau_{1}\right)} d\left(p, q, \tau_{1}\right)$ for $\tau \in\left[\tau_{1}, \tau_{2}\right]$ with $C$ denoting an upper bound for Ricci curvature. By this and the volume comparison, we infer that $d q$ grows at most like $e^{c_{1} d\left(p, q, \tau_{1}\right)}$ for a positive constant $c_{1}$. Hence (3.21) and (3.22) yield a finite upper bound for

$$
\int_{\tau_{1}}^{\tau_{2}} \int_{M}\left(|\nabla l| \cdot|\nabla \phi|+\left(\left|l_{\tau}\right|+|\nabla l|^{2}+|R|+\frac{n}{2 \tau}\right)|\phi|\right) d q d \tau
$$


In the case of a nonnegative curvature operator, we argue in a similar fashion, utilizing however different lemmas. Applying Theorem 2.18 we again infer (3.21). By Lemma 2.3 or Lemma 3.2, there exists a minimum point $x(\tau)$ of $l(\cdot, \tau)$ for each $\tau \in(0, T)$. By the Harnack inequality (2.57) in Theorem 2.18 and Lemma 3.1 we deduce

$$
l\left(x\left(\tau_{1}\right), \tau\right) \leq \frac{n}{2}\left(\frac{\tau_{2}}{\tau_{1}}\right)^{C}
$$

for $\tau \in\left[\tau_{1}, \tau_{2}\right]$. Then Lemma 3.2 leads to

$$
l(q, \tau) \geq \frac{C_{1}}{\tau_{2}} d^{2}\left(x\left(\tau_{1}\right), q, \tau\right)-\frac{n}{2}\left(\frac{\tau_{2}}{\tau_{1}}\right)^{C}-1
$$

for $\tau \in\left[\tau_{1}, \tau_{2}\right]$ and all $q \in M$. By volume comparison, $d q$ (at time $\tau$ ) grows at most at the Euclidean rate, i.e. $d\left(x\left(\tau_{1}\right), q, \tau\right)^{n-1}$, with $x\left(\tau_{1}\right)$ as the geodesic center. Hence (3.21) and (3.24) lead to a desired finite upper bound.

Part 2. The Integral Inequality

For each natural number $k$ we choose a smooth nonnegative function $\zeta_{k} \leq 1$ on the real line such that $\zeta_{k}=1$ on $[0, k], \zeta_{k}=0$ on $[k+2, \infty)$ and $\left|\zeta_{k}^{\prime}\right| \leq 1$ everywhere. In the case of bounded sectional curvature we define $\eta_{k}$ on $M \times\left[\tau_{1}, \tau_{2}\right]$ by the formula $\eta(q, \tau)=\zeta_{k}(d(p, q, \tau))$. Then we have $0 \leq \eta_{k} \leq 1,\left|\nabla \eta_{k}\right| \leq 1$ everywhere, $\eta_{k}(q, \tau)=1$ whenever $d(p, q, \tau) \leq k$, and $\eta_{k}(q, \tau)=0$ whenever $d(p, q, \tau) \geq k+2$. By Theorem 2.23 we then have

$$
\int_{\tau_{1}}^{\tau_{2}} \int_{M}\left\{\nabla l \cdot\left(\nabla \eta_{k} \phi+\eta_{k} \nabla \phi\right)+\left(l_{\tau}+|\nabla l|^{2}-R+\frac{n}{2 \tau}\right) \eta_{k} \phi\right\} d q d \tau \geq 0 .
$$

Let $I_{k}$ denote the integral $\int_{\tau_{1}}^{\tau_{2}} \int_{M}\left\{\nabla l \cdot \eta_{k} \nabla \phi+\left(l_{\tau}+|\nabla l|^{2}-R+\frac{n}{2 \tau}\right) \eta_{k} \phi\right\} d q d \tau$, and let $I$ denote the integral $\int_{\tau_{1}}^{\tau_{2}} \int_{M}\left\{\nabla l \cdot \nabla \phi+\left(l_{\tau}+|\nabla l|^{2}-R+\frac{n}{2 \tau}\right) \phi\right\} d q d \tau$. Then we have

$$
\left|I_{k}-I\right| \leq \int_{\tau_{1}}^{\tau_{2}} \int_{B_{k+2}(p, \tau)-B_{k}(p, \tau)}\left(|\nabla l \cdot \nabla \phi|+\left.\left|l_{\tau}+\right| \nabla l\right|^{2}-R+\frac{n}{2 \tau} \mid \phi\right) d q d \tau .
$$

By the above arguments for the absolute convergence in the case of bounded sectional curvature we infer

$$
\left|I_{k}-I\right| \leq \bar{C}_{1}\left(\tau_{2}-\tau_{1}\right) e^{-\bar{c}_{1} k}
$$

for some positive constants $\bar{C}_{1}$ and $\bar{c}_{1}$. Similarly, we have

$$
\left|\int_{\tau_{1}}^{\tau_{2}} \int_{M} \nabla l \cdot \nabla \eta_{k} \phi d q d \tau\right| \leq \int_{\tau_{1}}^{\tau_{2}} \int_{M-B_{k}(p, \tau)}|\nabla l| \phi d q d \tau \leq \bar{C}_{2}\left(\tau_{2}-\tau_{1}\right) e^{-\bar{c}_{2} k}
$$

for some positive constants $\bar{C}_{2}$ and $\bar{c}_{2}$.

In the case of a nonnegative curvature operator we set $\eta_{k}(q, \tau)=\zeta_{k}\left(d\left(x\left(\tau_{1}\right), q, \tau\right)\right)$ and use $x\left(\tau_{1}\right)$ as the geodesic center. Arguing as before we again obtain the estimates (3.27) and (3.28).

Taking the limit as $k \rightarrow \infty$ we arrive at the desired integral inequality.

\section{The REDUCED VOLume}

We continue with the solution $(M, g=g(\tau))$ of the backward Ricci flow on $[0, T)$ as before (assuming that $g(\tau)$ is complete for each $\tau \in[0, T)$ ). 
Definition 5. We define the reduced volume (of Perelman) $\tilde{V}(\tau)$ to be

$$
\tilde{V}(\tau)=\tilde{V}_{g}(\tau)=\int_{M} \tau^{-\frac{n}{2}} e^{-l(q, \tau)} d q .
$$

A basic property of $\tilde{V}$ is its invariance under the rescaling $g(\tau) \rightarrow g_{a}(\tau)=$ $a^{-1} g(a \tau)$, which easily follows from Lemma 2.2. Our main goal is to obtain monotonicity of the reduced volume and its upper bounds, and the associated rigidities. For this purpose, we need as in [P1] the following weighted monotonicity of the Jacobian of the $\mathcal{L}$-exponential map given in $[\mathrm{P} 1]$.

Lemma 4.1. Let $J(\tau)(v)=J_{g}(\tau)(v)$ denote the Jacobian of the $\mathcal{L}$-exponential map $\exp _{p}^{\mathcal{L}, \tau}$ at $v \in \Omega^{T_{p} M}(\tau)$, where $T_{p} M$ is equipped with the metric $g(\tau)_{p}$. Then we have

$$
\frac{d}{d \tau} \tau^{-\frac{n}{2}} e^{-l(v, \tau)} J(\tau)(v) \leq 0
$$

for each $v \in \Omega^{T_{p} M}(\tau)$, where $l(v, \tau)=l\left(\gamma_{v}(\tau), \tau\right) \quad\left(\gamma_{v}\right.$ is given in Definition 2). Moreover, if $\tau_{1}^{-\frac{n}{2}} e^{-l\left(v, \tau_{1}\right)} J\left(\tau_{1}\right)(v)=\tau_{2}^{-\frac{n}{2}} e^{-l\left(v, \tau_{2}\right)} J\left(\tau_{2}\right)(v)$ for $\tau_{1}<\tau_{2}$ and $v \in$ $\Omega^{T_{p} M}\left(\tau_{2}\right)$, then the equation

$$
\text { Ric }-\frac{1}{2 \tau} g+\nabla^{2} l=0
$$

holds true along $\gamma_{v}$ on the interval $\left[\tau_{1}, \tau_{2}\right]$.

Proof. This follows from the arguments on pages 16 and 17 in [P1].

Lemma 4.2. Consider $v \in \Omega(\hat{\tau})$ for some $\hat{\tau}$. Then

$$
\lim _{\tau \rightarrow 0} \tau^{-\frac{n}{2}} e^{-l(v, \tau)} J(\tau)(v)=e^{-\frac{|v|^{2}}{4}} .
$$

Consequently,

$$
\tau^{-\frac{n}{2}} e^{-l(v, \tau)} J(\tau)(v) \leq e^{-\frac{|v|^{2}}{4}}
$$

for each $\tau$.

Proof. Set $\tilde{J}(\tau)(v)=\tau^{-\frac{n}{2}} e^{-l(v, \tau)} J(\tau)(v)$. The following transformation formula is easy to verify:

$$
\tilde{J}_{g_{a}}(\tau)(a v) d v_{g_{a}(0)}=\tilde{J}(a \tau)(v) d v,
$$

where $g_{a}(\tau)=a^{-1} g(a \tau)$ and $a \tau \leq \hat{\tau}$. In particular,

$$
\tilde{J}_{g_{a}}(1)(a v) d v_{g_{a}(0)}=J(a)(v) d v .
$$

Using $\exp ^{\mathcal{L}, \hat{\tau}}$ we pull back $g, 0 \leq \tau \leq \bar{\tau}$ to $\Omega^{T_{p}}(\bar{\tau})$, and then pull it back by the scaling map $\Phi_{a}\left(v^{\prime}\right)=a v^{\prime}, v^{\prime} \in T_{p} M$. The resulting metrics will be denoted by $g^{*}$. Applying (4.7) to $g^{*}$ we deduce that

$$
\tilde{J}(a)(v)=\tilde{J}_{g_{a}^{*}}(1)(v)
$$

for $0<a<\hat{\tau}$. Next observe that over $[0,2]$, the $g_{a}^{*}$ converge smoothly on compact sets of $T_{p} M$ to the Euclidean steady soliton $g^{0}(\tau) \equiv g(0)_{p}$ as $a \rightarrow 0$. Moreover, the image of the minimal $\mathcal{L}$-geodesic from the reference point 0 to $v$ remains in a fixed compact set during the convergence, which follows from the arguments in the proof of Proposition 2.5. It follows that $\lim _{a \rightarrow 0} \tilde{J}(a)(v)=\tilde{J}_{g^{0}}(1)(v)=e^{-\frac{|v|^{2}}{4}}$.

The inequality (4.5) follows from (4.4) and Lemma 4.1. 
Theorem 4.3. Assume that the Ricci curvature is bounded from below on $[0, \bar{\tau}]$ for some $\bar{\tau}$. Then $\tilde{V}(\tau) \leq(4 \pi)^{\frac{n}{2}}$ for each $\tau \in(0, \bar{\tau}]$.

Proof. By Lemma 2.16 and Lemma 4.2 we have

$$
\tilde{V}(\tau)=\int_{\Omega^{T_{p}}(\tau)} \tau^{-\frac{n}{2}} e^{-l(v, \tau)} J(\tau)(v) d v \leq \int_{\Omega^{T_{p}}(\tau)} e^{-\frac{|v|^{2}}{4}} d v \leq \int_{T_{p} M} e^{-\frac{|v|^{2}}{4}} d v=(4 \pi)^{\frac{n}{2}} .
$$

Theorem 4.4. Assume that the Ricci curvature is nonnegative for $s \in[0, \tau]$. Then $\tilde{V}(\tau)<(4 \pi)^{\frac{n}{2}}$ unless $(M, g(0))$ is isometric to $\mathbf{R}^{n}$ and $g(s)=g(0)$ for all $s \in[0, \tau]$, in which case $\tilde{V}(\tau)=(4 \pi)^{\frac{n}{2}}$.

Proof. By (2.1), we have $\frac{\partial}{\partial t} d q=R d q$. This and (2.10) imply that

$$
\tilde{V}(\tau) \leq \tau^{-\frac{n}{2}} \int_{M} e^{-\frac{d^{2}(p, q, 0)}{4 \tau}} d q \leq\left.\tau^{-\frac{n}{2}} \int_{M} e^{-\frac{d^{2}(p, q, 0)}{4 \tau}} d q\right|_{0}
$$

By volume comparison, we have

$$
\left.\int_{M} e^{-\frac{d^{2}(p, q, 0)}{4 \tau}} d q\right|_{0} \leq \int_{\mathbf{R}^{n}} e^{-\frac{|x|^{2}}{4 \tau}} d x=(4 \pi \tau)^{\frac{n}{2}}
$$

Hence we arrive at the desired inequality. If $\tilde{V}(\tau)=(4 \pi)^{\frac{n}{2}}$, then $(4.11)$ must be an equality, and hence $(M, g(0))$ is isometric to $\mathbf{R}^{n}$. The second inequality in (4.10) must also be an equality. Consequently, $R \equiv 0$ and hence Ric $\equiv 0$ for $s \in[0, \tau]$. It follows that $g(s)=g(0)$ for $s \in[0, \tau]$.

Theorem 4.5. If the Ricci curvature is bounded from below on $[0, \tau]$ for each $\tau$, then $\tilde{V}(\tau)$ is a nonincreasing function.

Proof. By Lemma 2.16 we have

$$
\tilde{V}(\tau)=\int_{\Omega(\tau)} \tau^{-\frac{n}{2}} e^{-l(q, \tau)} d q=\int_{\Omega^{T_{p}}(\tau)} \tau^{-\frac{n}{2}} e^{-l(v, \tau)} J(\tau) d v
$$

where $d v$ denotes the Euclidean volume form on $T_{p} M$ determined by $g(\tau)_{p}$. By Lemma 2.10 we have for $\tau_{1}<\tau_{2}$ the inequality

$$
\tilde{V}\left(\tau_{2}\right)-\tilde{V}\left(\tau_{1}\right) \leq \int_{\Omega\left(\tau_{2}\right)} \tau_{2}^{-\frac{n}{2}} e^{-l\left(v, \tau_{2}\right)} J\left(\tau_{2}\right) d v-\int_{\Omega\left(\tau_{2}\right)} \tau_{1}^{-\frac{n}{2}} e^{-l\left(v, \tau_{1}\right)} J\left(\tau_{1}\right) d v .
$$

By Lemma 4.1 we then obtain the desired monotonicity.

Lemma 4.6. Assume either that the sectional curvature is bounded on $[0, \tau]$ for each $\tau$ or that the curvature operator is nonnegative. Then it follows that

$$
\tilde{V}\left(\tau_{2}\right)-\tilde{V}\left(\tau_{1}\right)=-\int_{\tau_{1}}^{\tau_{2}} \int_{M}\left(l_{\tau}-R+\frac{n}{2 \tau}\right) e^{-l} \tau^{-\frac{n}{2}} d q d \tau
$$

for all $0<\tau_{1}<\tau_{2}<T$.

Proof. We first assume a nonnegative curvature operator. Consider $\tau_{2}>\tau_{1}$. Applying Proposition 2.13 we deduce

$$
\begin{aligned}
\tilde{V}\left(\tau_{2}\right)-\tilde{V}\left(\tau_{1}\right) & =\int_{M} \int_{\tau_{1}}^{\tau_{2}} \frac{\partial}{\partial \tau}\left(\tau^{-\frac{n}{2}} e^{-l(q, \tau)} d q\right) \\
& =-\int_{M} \int_{\tau_{1}}^{\tau_{2}}\left(l_{\tau}-R+\frac{n}{2 \tau}\right) e^{-l} \tau^{-\frac{n}{2}} d q d \tau
\end{aligned}
$$


By the proof of Theorem 3.3, the last integral is absolutely convergent. Hence we can switch the integration order to arrive at the first equation in (4.13).

The proof of (4.13) in the case of bounded sectional curvature is similar. Note that in this case we also have

$$
\frac{\partial \tilde{V}}{\partial \tau}=\int_{M}\left(l_{\tau}-R+\frac{n}{2 \tau}\right) e^{-l} \tau^{-\frac{n}{2}}
$$

for every $\tau$. This can be seen by computing the relevant difference quotient and applying the dominated convergence theorem to pass to the limit.

We remark that (4.13) and Theorem 3.3 also imply the monotonicity of $\tilde{V}(\tau)$.

Definition 6. In this definition, let $g$ be a smooth solution of the backward Ricci flow on $N \times I$ for a smooth manifold $N$ and an interval $I$. We say that $g$ is a gradient shrinking soliton with time origin $\tau_{0}$ and potential function $f$ on an open subset $O$ of $N \times I$, where $f$ is a smooth function on $O$, provided that $g$ satisfies the gradient shrinking soliton equation

$$
\text { Ric }-\frac{1}{2\left(\tau-\tau_{0}\right)} g+\nabla^{2} f=0
$$

in $O$.

Lemma 4.7. Let $g$ be a gradient shrinking soliton on $N \times I$ with time origin $\tau_{0}$ and potential function $f$. Then $g$ evolves by the pullback of a family of diffeomorphisms coupled with scaling. More precisely, we have

$$
g(\tau)=\frac{\tau-\tau_{0}}{\bar{\tau}-\tau_{0}}\left(\phi^{-1}\right)^{*} g(\bar{\tau})
$$

where $\bar{\tau}$ is an arbitary point in $I$ and $\phi$ is the solution of the equation $\frac{\partial \phi}{\partial \tau}=\nabla f(\phi)$ with $\phi(\bar{\tau})=i d$ (id denotes the identity map of $N)$.

Proof. We have

$$
\frac{\partial \bar{g}}{\partial \tau}=2 R i c_{\bar{g}}=\frac{1}{\tau-\tau_{0}} \bar{g}-2 \nabla_{\bar{g}}^{2} f=\frac{1}{\tau-\tau_{0}} \bar{g}-L_{\nabla_{\bar{g}} f} \bar{g} .
$$

Hence

$$
\frac{\partial}{\partial \tau} \phi^{*} \bar{g}=\frac{1}{\tau-\tau_{0}} \phi^{*} \bar{g}
$$

The equation (4.17) follows.

Lemma 4.8. As in Definition 6, let $g$ be a smooth solution of the backward Ricci flow on $N \times I$. Let $f$ be a smooth function on an open subset $O$ of $N \times I$. We set $u=(4 \pi \tau)^{-\frac{n}{2}} e^{-f}$ and $v=\left[\tau\left(2 \nabla f-|\nabla f|^{2}+R\right)+f-n\right]$. Then we have

$$
\square v=-2 \tau\left|R i c+\nabla^{2} f-\frac{1}{\tau} g\right|^{2} u+2 \tau u \Delta\left(u^{-1} \square u\right),
$$

where $\square u=u_{\tau}-\nabla u+R u$. Consequently, if $u$ satisfies the heat equation $\square u=0$, or equivalently

$$
\frac{\partial f}{\partial \tau}-\Delta f+|\nabla f|^{2}-R+\frac{n}{2 \tau}=0,
$$

then $v$ satisfies the equation

$$
\square v=-2 \tau\left|R i c+\nabla^{2} f-\frac{1}{\tau} g\right|^{2} .
$$


In particular, if (4.21) holds, then $g$ is a gradient shrinking soliton on $O$ with time origin 0 and potential function $f$ if and only if $\square v=0$ in $O$.

Proof. This is a reformulation of [P1, Proposition 9.1]. The formula (4.20) follows from routine computations; see e.g. [KL].

Now we return to our previous $g$ on $M \times[0, T)$.

Theorem 4.9. Assume either that the sectional curvature is bounded on $[0, \tau]$ for each $\tau<T$, or that the curvature operator is nonnegative. Assume that $\tilde{V}\left(\tau_{1}\right)=$ $\tilde{V}\left(\tau_{2}\right)$ for some $\tau_{1}<\tau_{2}$. Then $l$ is smooth on $M \times\left(\tau_{1}, \tau_{2}\right)$ and $g$ is a gradient shrinking soliton on $M \times\left(\tau_{1}, \tau_{2}\right)$ with time origin 0 and potential function $l$.

Proof. Assume $\tilde{V}\left(\tau_{1}\right)=\tilde{V}\left(\tau_{2}\right)$ for some $\tau_{1}<\tau_{2}$. By Lemma 4.6 we have

$$
\int_{\tau_{1}}^{\tau_{2}} \int_{M}\left(l_{\tau}-R+\frac{n}{2 \tau}\right) e^{-l} \tau^{-\frac{n}{2}} d q d \tau=0 .
$$

We set

$$
Q_{\tau_{1}, \tau_{2}}(\phi)=\int_{\tau_{1}}^{\tau_{2}} \int_{M}\left\{\nabla l \cdot \nabla \phi+\left(l_{\tau}+|\nabla l|^{2}-R+\frac{n}{2 \tau}\right) \phi\right\} d q d \tau
$$

for admissible $\phi$, which are locally Lipschitz functions $\phi$ on $M \times\left[\tau_{1}, \tau_{2}\right]$ such that $|\phi| \leq C \tau^{-\frac{n}{2}} e^{-l}$ and $|\nabla \phi| \leq C \tau^{-\frac{n+1}{2}} \sqrt{l+\tau+1} e^{-l}$ for some bound factor $C>0$ depending on $\phi$. By Proposition 2.14, Proposition 2.19 and Theorem 2.18, the function $\tau^{-\frac{n}{2}} e^{-l}$ is admissible. By (4.23) we have $Q_{\tau_{1}, \tau_{2}}\left(\tau^{-\frac{n}{2}} e^{-l}\right)=0$. For an arbitrary nonnegative admissible $\phi$ with bound factor $C$ we have by Theorem $3.3, Q_{\tau_{1}, \tau_{2}}(\phi) \geq 0$ and $Q_{\tau_{1}, \tau_{2}}\left(C \tau^{-\frac{n}{2}} e^{-l}-\phi\right) \geq 0$, whence $0 \leq Q_{\tau_{1}, \tau_{2}}(\phi) \leq C Q_{\tau_{1}, \tau_{2}}\left(\tau^{-\frac{n}{2}} e^{-l}\right)=0$, i.e. $Q_{\tau_{1}, \tau_{2}}(\phi)=0$. By linearity of $Q_{\tau_{1}, \tau_{2}}$ we then infer that $Q_{\tau_{1}, \tau_{2}}(\phi)=0$ for all admissible $\phi$ (simply write $\phi$ as the sum of its positive and negative parts), in particular for all Lipschitz $\phi$ with compact support. The standard regularity theory for parabolic equations implies that $l$ is smooth on $M \times\left(\tau_{1}, \tau_{2}\right)$ and satisfies

$$
\frac{\partial l}{\partial \tau}-\Delta l+|\nabla l|^{2}-R+\frac{n}{2 \tau}=0 .
$$

By Lemma 2.22 we then also have

$$
2 \Delta l-|\nabla l|^{2}+R+\frac{l-n}{\tau}=0 .
$$

Now we can apply Lemma 4.8 with $f=l$. By (4.25), the equation (4.21) holds true. By $(4.26), v=0$. Hence we conclude that $g$ is a gradient shrinking soliton with time origin 0 and potential function $l$. (The implication of Lemma 4.8, i.e. $[\mathrm{P} 1,(9.1)]$, was first pointed out to us by G. Wei. Note that a similar argument is used in the proof of Theorem 10.1 in [P1].)

Theorem 4.10. Assume that the sectional curvature is bounded on $[0, \tau]$. Then $\tilde{V}(\tau)<(4 \pi)^{\frac{n}{2}}$ unless $(M, g(0))$ is isometric to $\mathbf{R}^{n}$ and $g(s)=g(0)$ for each $s \in$ $[0, \tau]$.

Proof. By Theorem 4.3, $\tilde{V}(\tau) \leq(4 \pi)^{\frac{n}{2}}$. Assume that the equality holds. By Theorem 4.5 and Theorem $4.9, g$ is a gradient shrinking soliton on $M \times(0, \tau)$ with time origin 0 and potential function $l$. By Lemma 4.7, $g\left(\tau^{\prime}\right)=\frac{\tau^{\prime}}{\tilde{\tau}} \phi^{*} g(\tilde{\tau})$ for $\tau^{\prime}, \tilde{\tau} \in(0, \tau)$. Since the sectional curvature is bounded, we can let $\tilde{\tau} \rightarrow 0$ to deduce that $g\left(\tau^{\prime}\right)$ is flat for each $\tau^{\prime} \in(0, \tau)$. The desired conclusion then follows from Theorem 4.4. 


\section{REFERENCES}

[F] H. Federer, Geometric Measure Theory, Springer-Verlag, 1969. MR0257325 (41:1976)

[GW] R. E. Greene and H. Wu, On the subharmonicity and plurisubharmonicity of geodesically convex functions, Indiana Univ. Math. J. 22 (1973), 641-653. MR0422686 (54:10672)

[H] R. Hamilton, Four manifolds with positive curvature operator, J. Diff. Geom. 24 (1986), 153-179. MR862046 (87m:53055)

[KL] B. Kleiner and J. Lott, Notes on Perelman's papers, www.math.1sa.umich.edu/research/ ricciflow/perelman.html

[P1] G. Perelman, The entropy formula for the Ricci flow and its geometric applications, arXiv:math.DG/0211159.

[P2] G. Perelman, Ricci flow with surgeries on three-manifolds, arXiv:math.DG/0303109.

[S] W. -X. Shi, Deforming the metric on complete Riemannian manifolds, J. Diff. Geom. 30 (1989), 223-301. MR1001277 (90i:58202)

[Y1] R. Ye, On the l-function and the reduced volume of Perelman.

[Y2] R. Ye, On the l-function and the reduced volume of Perelman II, this issue.

[Y3] R. Ye, Notes on convex functions on Riemannian manifolds.

Department of Mathematics, University of California, Santa Barbara, California 93106

E-mail address: yer@math.ucsb.edu 